\title{
2 Stochastic Oblique Impact on Composite Laminates: A Concise Review 3 and Characterization of the Essence of Hybrid Machine Learning 4 Algorithms
}

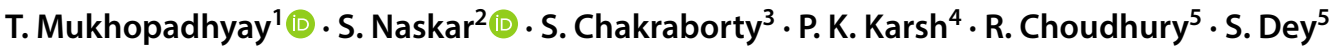

Received: 21 September 2019/ Accepted: 29 April 2020

(c) CIMNE, Barcelona, Spain 2020

\begin{abstract}
Due to the absence of adequate control at different stages of complex manufacturing process, material and geometric properties of composite structures are often uncertain. For a secure and safe design, tracking the impact of these uncertainties on the structural responses is of utmost significance. Composite materials, commonly adopted in various modern aerospace, marine, automobile and civil structures, are often susceptible to low-velocity impact caused by various external agents. Here, along with a critical review, we present machine learning based probabilistic and non-probabilistic (fuzzy) low-velocity impact analyses of composite laminates including a detailed deterministic characterization to systematically investigate the consequences of source- uncertainty. While probabilistic analysis can be performed only when complete statistical description about the input variables are available, the non-probabilistic analysis can be executed even in the presence of incomplete statistical input descriptions with sparse data. In this study, the stochastic effects of stacking sequence, twist angle, oblique impact, plate thickness, velocity of impactor and density of impactor are investigated on the crucial impact response parameters such as contact force, plate displacement, and impactor displacement. For efficient and accurate computation, a hybrid polynomial chaos based Kriging (PC-Kriging) approach is coupled with in-house finite element codes for uncertainty propagation in both the probabilistic and non- probabilistic analyses. The essence of this paper is a critical review on the hybrid machine learning algorithms followed by detailed numerical investigation in the probabilistic and non-probabilistic regimes to access the performance of such hybrid algorithms in comparison to individual algorithms from the viewpoint of accuracy and computational efficiency.
\end{abstract}

\section{Introduction}

T. Mukhopadhyay, S. Naskar, S. Chakraborty, P. K. Karsh, R. Choudhury and S. Dey have contributed equally to this work.

T. Mukhopadhyay

tanmoy@iitk.ac.in

S. Naskar

susmitanaskar@iitb.ac.in

1 Department of Aerospace Engineering, Indian Institute of Technology Kanpur, Kanpur, India

2 Department of Aerospace Engineering, Indian Institute of Technology Bombay, Mumbai, India

3 Department of Applied Mechanics, Indian Institute of Technology Delhi, New Delhi, India

4 Department of Mechanical Engineering, Parul Institute of Engineering and Technology, Parul University, Vadodara, India

5 Mechanical Engineering Department, National Institute of Technology Silchar, Silchar, India
Due to the high specific strength, stiffness, rigidity, fatigue, corrosion resistance and other outstanding mechanical characteristics (with tunable characteristics) compared to standard metallic structural materials, laminated composite plates have a broad application in the spacecraft, marine, automotive, mechanical and civil sectors. Composite structures are often susceptible to low-velocity impact caused by various external agents, leading to a significant influence on the intended performance of the system. Therefore, investigating the behaviour of composite structures subjected to impact load is of utmost importance. On the other hand, uncertainties in a composite material may arise due to presence of voids in between the laminate, incomplete knowledge about the fibre parameters, porosity, alternation in ply thickness and various other inevitable issues involved in the complex manufacturing process. Quite naturally, the low-velocity impact responses are affected by the presence 
of these uncertainties. A general overview of the sources of uncertainty in the computational framework of a structural system is presented in Fig. 1 [1]. One ad-hoc way to deal with these uncertainties is to introduce the so- called partial safety factors at the design stage. However, a more rigorous method will demand quantification of the effect of the material and the geometric uncertainties on the output responses. To this end, we would pursue both probabilistic and non-probabilistic low-velocity impact assessment of composite laminates to cover two possible instances of getting an adequate statistical report on the input parameters, or unavailability of the same owing to restrictions on performing experiments involving a large number of samples.

Researchers, over the years, have studied the behaviour of composite structures under the action of impact load. While $\mathrm{Xu}$ and Chen [2] conducted low-velocity impact analysis of carbon- epoxy laminates for damage detection, Liu et al. [3] studied the influence of shape of impactor (such as conical, hemispherical and flat) on the low-velocity impact responses of sandwich plate. In both cases, experimental as well as numerical analyses were performed. Jagtap et al. [4] carried out finite element (FE) simulation for damage identification of laminated plates due to impact loading.
The effect of boundary condition and velocity of impactor were determined. Similarly, Balasubramani et al. [5] performed numerical investigation to determine the effect of boundary conditions, the thickness of laminate, impactor's mass and velocity on transverse and longitudinal stress of the composite laminate due to low-velocity impact loading. Tan and Sun [6] and Sun and Chen [7] also used the finite element method with Newmark time integration scheme to investigate low-velocity impact on composite structures. A comprehensive review on low-velocity impact loading on composite structures can be found in [8]. Ahmed and Wei [9] also reviewed numerical and experimental methods for computing dynamic and static responses of composite plates subjected low-velocity impact and quasi-static loads.

Several works dealing with failure mechanism of composite plates subjected to low-velocity impact load can be found in the literature. While Yuan et al. [10] used an analytical model based on the theory of first order shear deformation for the analysis of damage and deformation of laminated glass under low-velocity impact, Zhang and Zhang [11] applied FE model for damage detection in composite structures due to low-velocity impact. Feng and Aymerich [12], Maio et al. [13] and Kim et al. [14] developed and applied

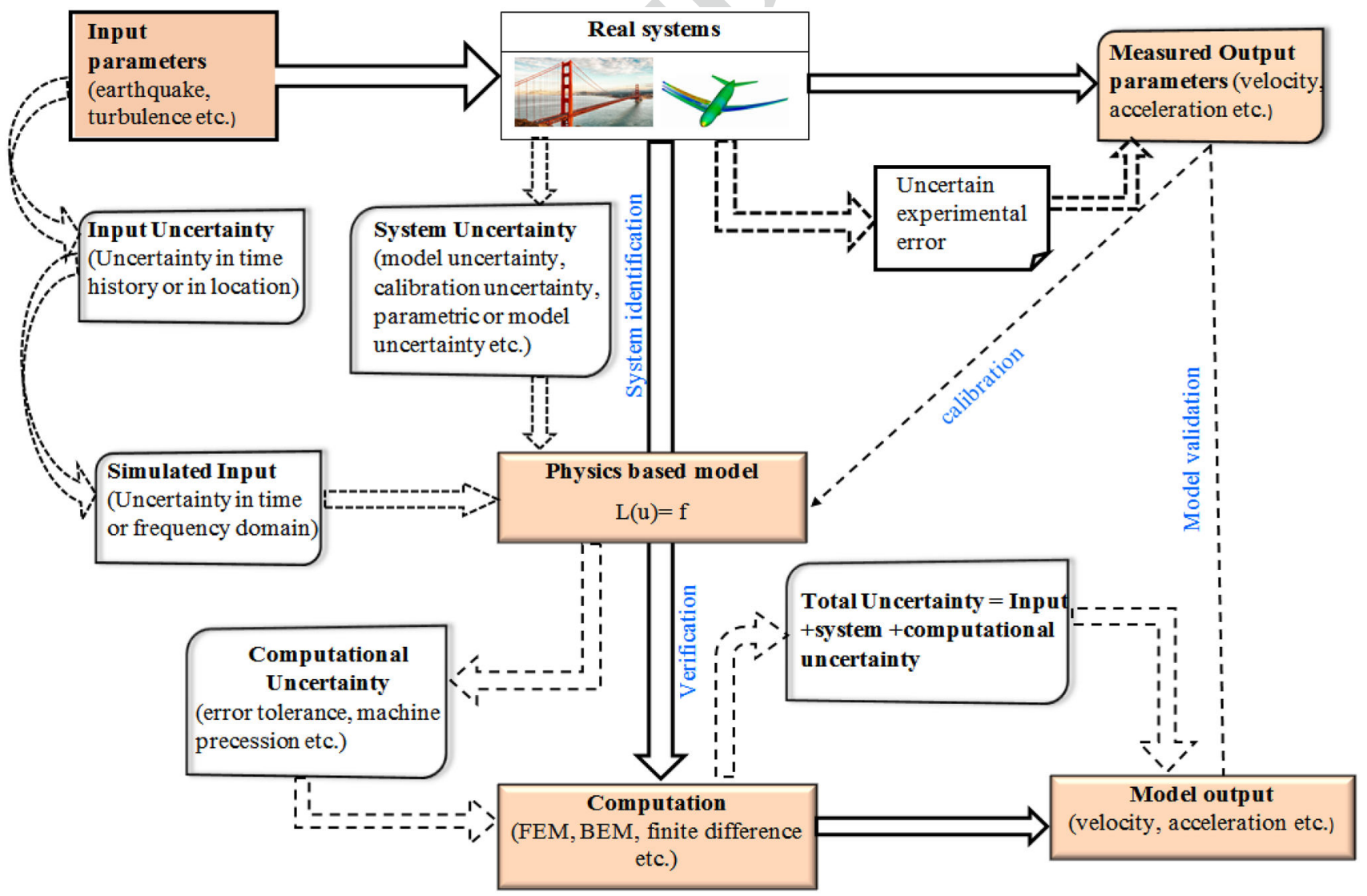

Fig. 1 General overview of the sources of uncertainty in the computational framework of a structural system 
progressive damage models to investigate the failure mechanism of laminated composite due to the low-velocity impact. Lipeng et al. [15] investigated delamination failure due to impact load by using a self-adapting delamination element method. Johnson et al. [16] presented different models for failure analysis of composite plates by considering internal damage and delamination due to impact loading. Coutellier et al. [17] developed a model for delamination detection in thin composite structures. Jih and Sun [18], on the other hand, investigated experimentally the delamination in laminated composite plates due to low-velocity impact.

Despite the vast literature on low-velocity impact analysis of composite structures, none of these studies consider the presence of uncertainties in the system. Due to the complexity of manufacturing, accurate design specifications of composite structures cannot be achieved in real life. As a consequence, uncertainties in a composite structure are unavoidable. In composite material, the main sources of uncertainties are due to variation in material properties and inaccurate geometrical properties. Such uncertainties are introduced in the elementary input level (elemental mass and stiffness matrix), and propagate to the global level (global mass and stiffness matrix) of composite structures and hence, leads to a significant deviation from the deterministic value of impact responses. In the present paper, the effects such source-uncertainties on the lowvelocity oblique impact (refer to Fig. 2a) response of composite plates are aimed to be addressed. The analysis is divided into three sections namely deterministic, probabilistic and nonprobabilistic, the later two sections being dedicated to stochastic analysis and uncertainty quantification (UQ). Only when the probabilistic distributions of uncertain input parameters are accessible can the probabilistic analyses be performed. In many instances though, it is not possible to obtain the complete probabilistic distributions of the input variables. In such cases, non-probabilistic fuzzy analysis can be employed to portray the effects of uncertainty. It is to be noted that both conventional probabilistic and non-probabilistic analysis techniques involve significant computational efforts due to the requirement of performing thousands of expensive finite element simulations. One way to circumvent this issue is to develop a machine learning model on the basis of representative original finite element simulations. It is worthy to note here that machine learning is a broad domain. A schematic diagram showcasing the various aspects of machine learning techniques and its relationship with data science is shown in Fig. 3. In this work, we are only interested in supervised learning techniques. Popular supervised learning techniques include Gaussian process or Kriging [19-22], Polynomial chaos expansion (PCE) [23-25], analysis-of-variance decomposition [26-29], Polynomial chaos based Kriging (PC- Kriging) [30-33] etc. In this work, we review three machine learning techniques in the context of stochastic low-velocity impact analysis. The machine learning techniques reviewed here are polynomial chaos expansion, Kriging and polynomial chaos based Kriging.

This paper is composed of six sections in the order of chronological inter-dependence including the current introduction section. Section 2 describes governing equations for the analysis of the transient low-velocity oblique impact of composite plates that includes the descriptions of dynamic equations, contact law and Newmark's integration scheme. In Sect. 3, detailed description of the surrogate model based on PC-Kriging is provided. Section 4 provides both probabilistic and non-probabilistic stochastic approaches for the impact analysis of low-velocity. The numerical results are presented in Sect. 5 (deterministic, probabilistic and fuzzy based nonprobabilistic results including the comparative performance of three different surrogate models i.e. PCE, Kriging and PCKriging). Finally, in Sect. 6, major observations and conclusion are provided along with an overview of the current level of development in relevant research fields.

\section{Review of the Governing Equations for Low-Velocity Impact on Laminated Composites}

A laminated composite plate is considered with length $L$, width $b$, and thickness $t$ subjected to normal and oblique impact loading (as shown in Fig. 2). The dynamic equation [34] of such system can be expressed as

$[M(\tilde{\zeta})]\{\ddot{\delta}\}+[K(\tilde{\zeta})]\{\delta\}=\{F(\tilde{\zeta})\}$

where $M(\tilde{\zeta}),(\tilde{\zeta}), \delta$ and $\ddot{\delta}$ are the randomized mass matrix, randomized stiffness matrix, displacement vector and acceleration vector, respectively, while $\{F\}$ is externally applied force vector. Here, $(\tilde{\zeta})$ indicates the degree of randomization. The force vector including the contact force $\left(F_{C}\right)$ in case of impact can be expressed as

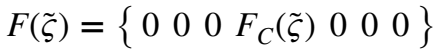

The equation of motion for the rigid impactor is given by

$m_{\text {imp }}(\tilde{\zeta}) \ddot{\delta}_{i m p}+F_{c}(\tilde{\zeta})=0$

where $m_{i m p}(\tilde{\zeta})$ is the mass of impactor while $\ddot{\delta}_{\text {imp }}$ is the acceleration of impactor.

\subsection{Contact Law}

Modified Hertzian contact law can be utilized to calculate the contact force between impactor and the composite plate [35]. The impactor is assumed as a spherical elastic solid body.

The contact force can be obtained during loading as 


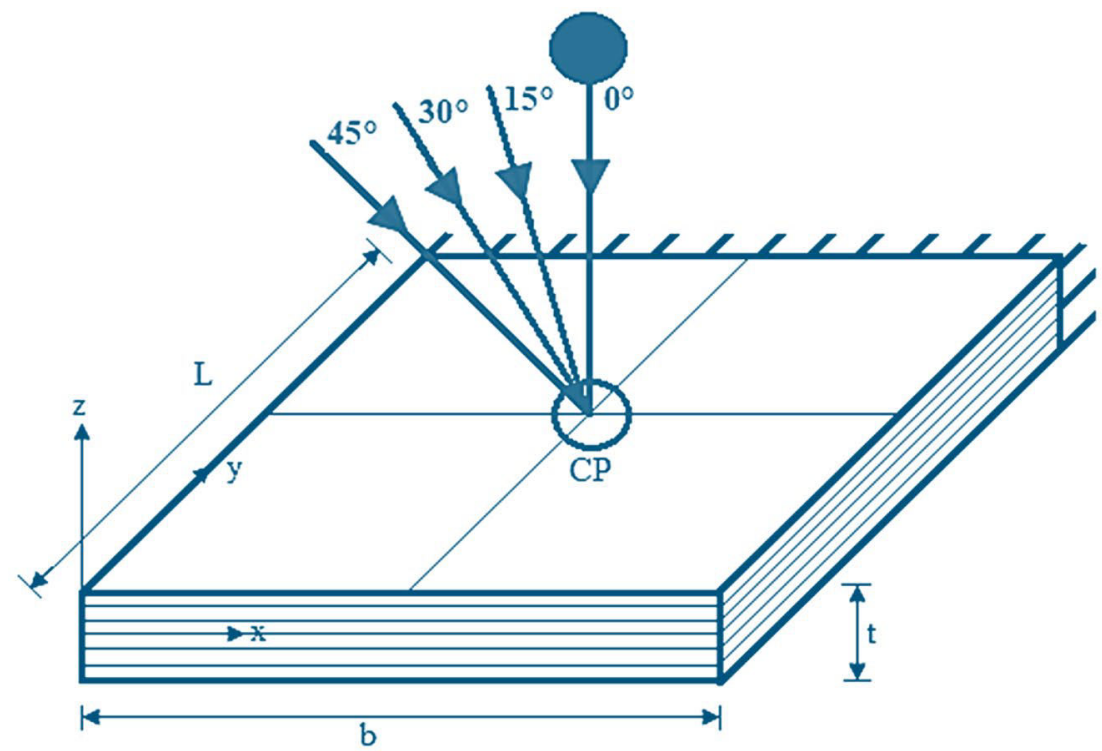

(a)
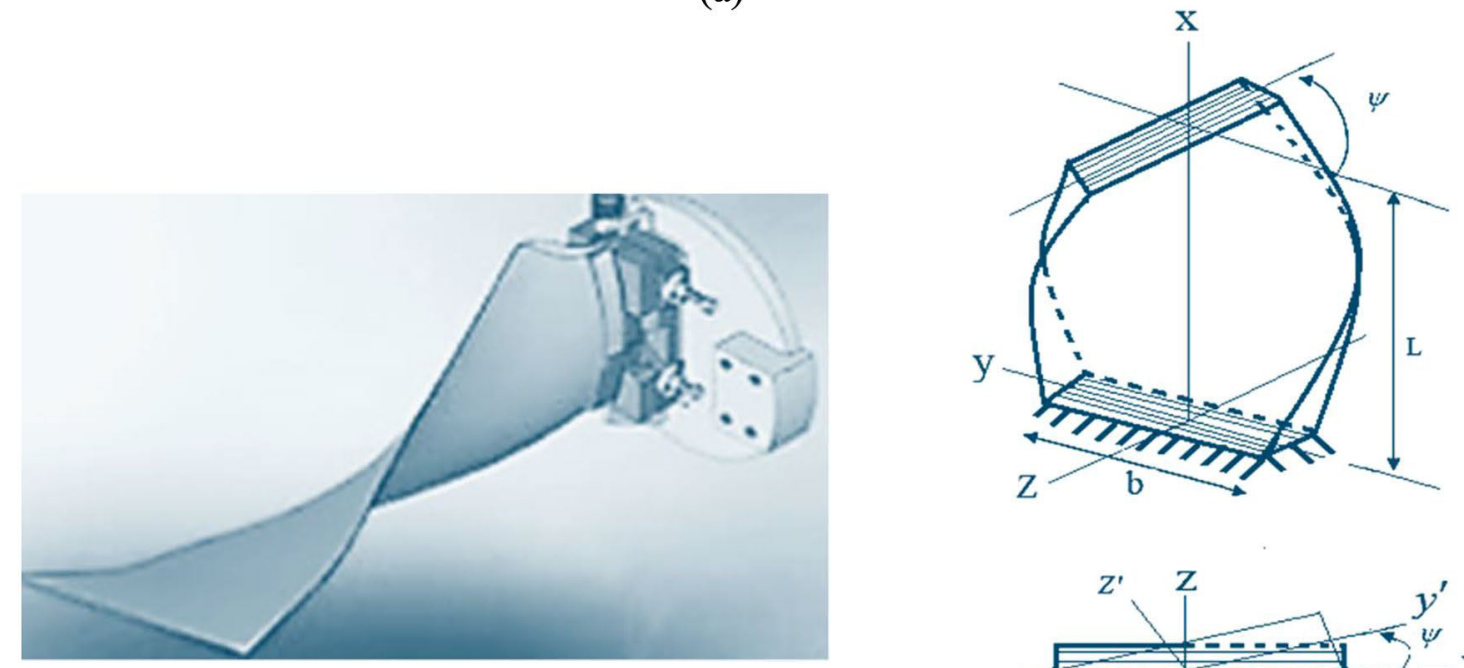

(b)

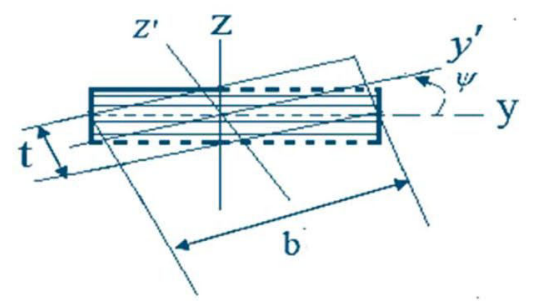

(c)

Fig. 2 a Laminated composite plate subjected to normal and oblique impact load by a spherical mass. b A typical example of twisted plate. c Geometric details of twist in the plate 
Fig. 3 Different facets of machine learning techniques

$F_{c}(\tilde{\zeta})=k(\tilde{\varsigma}) \gamma(\tilde{\zeta})^{1.5} \quad 0 \leq \gamma \leq \gamma_{m}$

where $\gamma$ denotes the local indentation and $k$ is the modified contact stiffness [36] which can be expressed by contact theory as

$k(\tilde{\varsigma})=\frac{4}{3} \sqrt{R_{i m p}} \frac{1}{\frac{\left[1-v_{i}^{2}(\tilde{\varsigma})\right]}{E_{i}(\tilde{\varsigma})}+\frac{1}{E_{y y}(\tilde{\zeta})}}$

where $E_{i}$ is the elastic modulus of the impactor, $E_{y y}$ is the elastic modulus of laminated composite plate of the uppermost ply in the transverse direction, while $R_{\text {imp }}$ and $v$ are the radius and Poisson's ratio of impactor, respectively. At the time of loading and unloading the contact force $\left(F_{C}\right)$ can be estimated as

$F_{c}(\tilde{\varsigma})=F_{m}\left[\frac{\gamma(\tilde{\varsigma})-\gamma_{0}}{\gamma_{m}-\gamma_{0}}\right]^{5 / 2}$ and $F_{c}(\tilde{\zeta})=F_{m}\left[\frac{\gamma(\tilde{\varsigma})-\gamma_{0}}{\gamma_{m}-\gamma_{0}}\right]^{3 / 2}$

where $F_{m}$ and $\gamma_{m}$ are the maximum contact force and maximum indentation, respectively. The permanent indentation $\left(\gamma_{0}\right)$ in loading and unloading cylce is given by

$\gamma_{0}=0 \quad$ when $\gamma_{m}<\gamma_{C r}$

$\gamma_{0}=\beta_{c}\left(\gamma_{m}-\gamma_{C r}\right) \quad$ when $\gamma_{m} \geq \gamma_{C r}$

where $\beta_{c}$ is the constant, while $\gamma_{C r}$ is the critical identation. For the oblique impact, the local indentaion is given by

$\gamma(t)(\tilde{\varsigma})=\gamma_{i m p}(t)(\tilde{\varsigma}) \cos \beta+\gamma_{p l t}\left(x_{c}, y_{c}, t\right)(\tilde{\varsigma}) \cos \psi$

where $\gamma_{i m p}$ and $\gamma_{p l t}$ are impactor's displacement and targeted plate displacements, respectively, while $\beta$ and $\psi$ are the oblique impact angle and twist angle, respectively, along the global z-direction, respectively. The contact force elements 21 . at the global direction of contact point can be described as

$F_{i x}=0, \quad F_{i y}=F_{c}(\tilde{\varsigma}) \sin \psi, \quad F_{i z}=F_{c}(\tilde{\varsigma}) \cos \psi$

\subsection{Newmark's Time Integration Scheme}

The contact force involved in the equilibrium Eqs. (1) and (3) is generally transient in nature for the dynamic response of a laminated composite plate under the impact by a spherical impactor. The time integration scheme of Newmark [37] is used to solve the equations that depend on time. Use of above scheme with time interval $\Delta t$ gives the subsequent relations at the time $t+\Delta t$

$[\bar{K}] \delta^{(t+\Delta t)}=\{\bar{F}\}^{(t+\Delta t)}$

$k_{i m p} \delta_{i m p}^{(t+\Delta t)}=\left\{F_{C}\right\}^{(t+\Delta t)}$

where $[\bar{K}]$ and $[\bar{k}]$ are the effective stiffness matrix of the plate and impactor, respectively, and given by

$[\bar{K}]=K+a_{0} M$

$[\bar{k}]=a_{0} m_{i m p}$

Effective contact forces at time $t+\Delta t$ can be derived as

$\{F\}^{(t+\Delta t)}=\{F\}^{(t+\delta t)}+[M]\left(a_{0} \delta^{(t)}+a_{1} \dot{\delta}^{(t)}+a_{2} \ddot{\delta}^{(t)}\right)$

$\bar{F}_{C_{e}}^{(t+\Delta t)}=F_{C}^{(t+\Delta t)}+m_{i m p}\left(a_{0} \delta_{i m p}^{(t)}+a_{1} \dot{\delta}_{i m p}^{(t)}+a_{2} \ddot{\delta}_{i m p}^{(t)}\right)$ 
The acceleration and velocity can be derived from displacement at time $t+\Delta t$ as

$$
\begin{aligned}
& \{\ddot{\delta}\}^{(t+\Delta t)}=a_{0}\left(\{\delta\}^{(t+\Delta t)}-\{\delta\}^{(t)}\right)-a_{1}\{\dot{\delta}\}^{(t)}-a_{2}\{\ddot{\delta}\}^{(t)} \\
& \ddot{\delta}_{i m p}^{(t+\Delta t)}=a_{0}\left(\delta_{i m p}^{(t+\Delta t)}-\delta_{i m p}^{(t)}\right)-a_{1} \dot{\delta}_{i m p}^{(t)}-a_{2} \ddot{\delta}_{i m p}^{(t)} \\
& \{\dot{\delta}\}^{(t+\Delta t)}=\{\dot{\delta}\}^{(t)}+a_{3}\{\ddot{\delta}\}^{(t)}+a_{4}\{\ddot{\delta}\}^{(t+\Delta t)} \\
& \dot{\delta}_{i m p}^{(t+\Delta t)}=\dot{\delta}_{i m p}^{(t)}+a_{3} \ddot{\delta}_{i m p}^{(t)}+a_{4} \ddot{\delta}_{i m p}^{(t+\Delta t)}
\end{aligned}
$$

The initial boundary condition considered as

$$
\delta=\dot{\delta}=\ddot{\delta}=0, \quad \delta_{i m p}=\ddot{\delta}_{i m p}=0 \text { and } \dot{\delta}_{i m p}=V_{0}
$$

where $V_{0}$ is the initial velocity of the impactor. The time integration constants can be expressed as

$a_{0}=\frac{1}{\beta^{\prime \prime} \delta t^{2}}, \quad a_{1}=\frac{1}{\beta^{\prime \prime} \Delta t}, \quad a_{2}=\frac{1}{2 \beta^{\prime \prime}}-1$,

$a_{3}=\left(1-\alpha^{\prime \prime}\right) \Delta t \quad$ and $\quad a_{4}=\alpha^{\prime \prime} \Delta t$

For the present study, the value of $\alpha^{\prime \prime}$ and $\beta^{\prime \prime}$ are considered as 0.5 and 0.25 , respectively.

\section{Hybrid Machine Learning Based on Kriging and PCE}

Let, $\boldsymbol{x}=\left\{x_{1}, \ldots, x_{N}\right\} \in \mathbb{R}^{N}$ to be the input variables and $\boldsymbol{y} \in \mathbb{R}^{O}$ to be the output responses. We also assume $\mathcal{M}(\cdot)$ to be the computational model (FE model in present case) such that

$y=\mathcal{M}(\boldsymbol{x})$

For impact analysis, the model $\mathcal{M}(\cdot)$ is computationally expensive to evaluate and hence, the task of quantifying the uncertainties in the output response $\boldsymbol{y}$ becomes difficult. One way to deal with this issue is to replace the computationally expensive finite element model $\mathcal{M}(\cdot)$ with a surrogate $\overline{\mathcal{M}}(\cdot)$. It can be noted here that we have used the words surrogate modelling and machine learning in identical sense keeping in mind its purpose in the context of this article. We would review three methods of machine learning in this section that can be used as a surrogate of the original simulation model.

\subsection{Polynomial Chaos Expansion}

Polynomial chaos expansion (PCE) is one of the most popular methods available in literature. This was first implemented by Wiener [38] and hence, is also known as 'Wiener Chaos expansion'. Xiu and Karniadakis [23] subsequently generalized the technique and proved its effectiveness for different continuous and discrete systems from the so called
Askey-scheme, $\mathcal{L}_{2}$ convergence in the corresponding Hilbert space.

Assuming $\mathbf{i}=\left(i_{1}, i_{2}, \ldots, i_{N}\right) \in \mathbb{N}_{0}^{N}$ to be a multi-index with $|\mathrm{i}|=i_{1}+i_{2}+\cdots+i_{N}$, and let $n \geq 0$ be an integer. The $n$th order PCE of $g(\boldsymbol{X})$ is given as:

$\hat{g}(\boldsymbol{X})=\sum_{|\mathrm{i}|=0}^{n} a_{\mathrm{i}} \boldsymbol{\Phi}_{\mathrm{i}}(\boldsymbol{X})$

where $\left\{a_{\mathbf{i}}\right\}$ are unknown coefficients that must be determined. $\Phi_{\mathrm{i}}(\boldsymbol{X})$ are $N$-dimensional orthogonal polynomials with maximum order of and satisfies

$E\left(\Phi_{\mathrm{i}}(X) \Phi_{\mathrm{j}}(X)\right)=\int_{\Omega} \Phi_{\mathrm{i}}(X) \Phi_{\mathrm{j}}(X) \varpi(x)=\delta_{i j}, \quad 0 \leq|\mathrm{i}|, \quad|\mathrm{j}| \leq N$

Here, $\delta_{\mathrm{ij}}$ denotes the multivariate kronecker delta function. It is to be noted that the orthogonal polynomials are dependent on the PDF $\varpi(x)$ of input variables. Table 1 presents the orthogonal polynomial type and the random variable type correspondence [23].

Over last two decades, researchers have developed and utilized different variants of PCE. Xiu and Karniadakis [23] proposed the Wiener-Askey PCE where the unknown coefficients associated with the coefficients were determined by using either collocation method or the Galerkin projection. With this method, it is possible to solve stochastic partial differential equations in an efficient way. However, Wiener-Askey PCE is intrusive in nature and hence, knowledge about the governing partial differential equation of the system is required. As a consequence, this method is not applicable to cases where the user only have some data and no knowledge about the process from which the data is generated.

To tackle the above-mentioned problem, researchers focused on developing nonintrusive (data-driven) PCE. The easiest and most popular way to train a data-driven PCE is by minimizing the least square error of the system

Table 1 The Correspondence of the type of orthogonal polynomial with distribution pattern

\begin{tabular}{llll}
\hline Type & Random variables & $\begin{array}{l}\text { Type of orthogo- } \\
\text { nal polynomial }\end{array}$ & Support \\
\hline Continuous & Gaussian & Hermite & $(-\infty, \infty)$ \\
& Gamma & Laguerre & {$[0, \infty)$} \\
& Beta & Jacobi & {$[a, b]$} \\
& Uniform & Legendre & {$[a, b]$} \\
Discrete & Poisson & Charlier & $\{0,1, \ldots\}$ \\
& Binomial & Krawtchouk & $\{0,1, \ldots, N\}$ \\
& Negative binomial & Meixner & $\{0,1, \ldots\}$ \\
& Hypergeometric & Hahn & $\{0,1, \ldots, N\}$ \\
\hline
\end{tabular}


$[39,40]$. However, this method is susceptible to overfitting and as a result often performs poorly. Methods for training a PCE model by using the quadrature rule can also be found in the literature $[41,42]$. However, both these training algorithms suffer from the curse of dimensionality and hence, are only applicable to small-scale problems with limited number of input variables.

To address the curse of dimensionality associated with least-square and quadrature based training algorithms, Blatman and Sudret [24, 43] two adaptive sparse PCEs which can used for solving problems having hundreds of input variables. Both the methods proposed follow similar flow where an iterative algorithm is used to determine the importance of the terms involved in PCE and the lesser important terms are removed. In the first method, the important terms in PCE are determined by tracking the change in coefficient of determination ${ }^{2}$ (due to addition/ removal of a term). In the second approach, a more rigorous framework, referred to as the least-angle regression is used to determine the important terms of PCE. With both these approaches, there is a significant reduction in the number of unknown coefficients associated with PCE and thereby, issues with hundreds of input variables can be solved.

Jacquelin et al. [44] identified that for lightly damped systems, the convergence of PCE is very poor. It was proposed that integrating Aitken's transformation into the framework of PCE can improve its convergence significantly. Pascual and Adhikari [45] hybridized the basic formulation of PCE by coupling it with perturbation method. Four variants of the hybrid perturbation-PCE was proposed and reduced spectral method was used to identify unknown coefficients associated with the bases. The proposed approaches were utilized to solve the stochastic eigenvalue problem. It was observed that the approaches proposed lead to a better approximation of larger eigenvalues.

\subsection{Kriging}

In today's time, one of the most popular machine learning technique is perhaps the Gaussian process, a.k.a. Kriging is a Bayesian machine learning technique where we assume that the response $y$, conditioned on input $x$ is a sample from a Gaussian process.

$y \mid x ; \mathbf{B}, \sigma, \boldsymbol{\theta} \sim \mathcal{G P}\left(\mu(x ; \mathbf{B}), \sigma^{2} R\left(x_{1}, x_{1} ; \boldsymbol{\theta}\right)\right)$

where $\mu(\cdot ; \mathbf{B})$ is the mean function and $R(\cdot, \cdot ; \boldsymbol{\theta})$ is the correlation kernel. $\mathbf{B}, \sigma$ and $\boldsymbol{\theta}$ are the hyperparameters of the Gaussian process respectively, denotes the unknown coefficients related to the mean function, the process variance and the length-scale parameter associated with the correlation kernel. In order to use Gaussian process as a machine learning technique, the hyperparameters needs to be estimated based on some training data. This can either be achieved by maximizing the likelihood [21] or by using the Bayes rule [46-49].

The most popular form of Gaussian process is the zero mean Gaussian process or the simple Kriging. In this variant, we assume $\mu(\cdot ; \mathbf{B})=0$. As a consequence, only $\sigma$ and $\theta$ are the only hyperparameters associated with the system. An improvement to the simple Kriging is the ordinary Kriging where we assume the mean function is assumed to be constant, $\mu(\cdot ; \mathbf{B})=a_{0}$ where $a_{0}$ is a constant. Unfortunately, the fact that the mean function is modelled as a constant often results in erroneous models.

To enhance the Kriging model's precision, universal Kriging was developed [50, 51]. In universal Kriging, the mean function represented as a linear regression model by using multivariate polynomials

$\mu(\cdot, \mathbf{B})=\sum_{i=1}^{P} a_{i} b_{i}(x)$

where $b_{i}(x)$ represents the $i$ th basis function and $a_{i}$ denotes the coefficient associated with the $i$ th basis function. With this setup, the mean function captures the largest variance in the data and the correlation function interpolates the residual. Considering, $\boldsymbol{x}=\left\{x^{1}, x^{2}, \ldots, x^{n}\right\}$ to be input samples and $g=\left\{g_{1}, g_{2}, \ldots, g_{n}\right\}$ to be the responses, the design matrix and the correlation matrix can be represented. The regression portion can be written as a $n \times p$ model matrix $F$,

$F=\left(\begin{array}{ccc}b_{1}\left(x^{1}\right) & \ldots & b_{p}\left(x^{1}\right) \\ \vdots & \ddots & \vdots \\ b_{1}\left(x^{n}\right) & \cdots & b_{p}\left(x^{n}\right)\end{array}\right)$

whereas the stochastic process is defined using a $n \times n$ correlation matrix $\Psi$

$\psi=\left(\begin{array}{ccc}\psi\left(x^{1}, x^{1}\right) & \ldots & \psi\left(x^{1}, x^{n}\right) \\ \vdots & \ddots & \vdots \\ \psi\left(x^{n}, x^{1}\right) & \cdots & \psi\left(x^{n}, x^{n}\right)\end{array}\right)$

where $\psi(\bullet, \bullet)$ is a correlation function, parameterised by a set of hyperparameters $\theta$. As already stated, the hyperparameters are identified either by using maximum likelihood estimation (MLE) or by using the Bayes rule.

Similar to PCE discussed in previous section, universal Kriging also suffers from the curse of dimensionality. To address this issue, blind Kriging was proposed in [51-54]. In blind Kriging, the polynomial order used to represent the mean function of the Gaussian process is selected in an adaptive manner. Bayes rule is used to training the blind Kriging model. It is worthwhile to mention that blind Kriging satisfies both the hierarchy criterion and the heredity criterion. As per the hierarchy criterion, 
lower order effects in the mean function are selected before the higher order effects. Whereas, as per the heredity criterion, an effect can only be important if its parent effects are already important. Other variants of Kriging includes Co-Kriging [55] and stochastic Kriging [56-58]. A comparative assessment from the viewpoint of accuracy and computational efficiency can be found in ref [19], where both high and low dimensional input parameter space was considered for a comprehensive analysis.

The choice of suitable correlation function is a crucial element for all the Kriging variants [59-61]. Correlation function that are commonly used with Gaussian process are mostly stationary and hence,

$\psi\left(x, x^{\prime}\right)=\prod_{j} \psi_{j}\left(\theta, x_{i}-x_{i}^{\prime}\right)$

With such as correlation function, it is possible to represent multivariate functions as product of one-dimensional correlations. Popular stationary correlation functions includes: (a) exponential correlation function (b) generalised exponential correlation function (c) Gaussian correlation function (d) linear correlation function (e) spherical correlation function (f) cubic correlation function and (g) spline correlation function. The mathematical forms of all the correlation functions are provided below:

1. Exponential correlation function:

$$
\psi_{j}\left(\theta ; d_{j}\right)=\exp \left(-\theta_{j}\left|d_{j}\right|\right)
$$

2. Generalised exponential correlation function:

$$
\psi_{j}\left(\theta ; d_{j}\right)=\exp \left(-\theta_{j}\left|d_{j}\right|^{\theta_{n+1}}\right), \quad 0<\theta_{n+1} \leq 2
$$

3. Gaussian correlation function:

$$
\psi_{j}\left(\theta ; d_{j}\right)=\exp \left(-\theta_{j} d_{j}^{2}\right)
$$

4. Linear correlation function:

$$
\psi_{j}\left(\theta ; d_{j}\right)=\max \left\{0,1-\theta_{j}\left|d_{j}\right|\right\}
$$

5. Spherical correlation function:

$$
\psi_{j}\left(\theta ; d_{j}\right)=1-1.5 \xi_{j}+0.5 \xi_{j}^{2}, \xi_{j}=\min \left\{0, \theta_{j}\left|d_{j}\right|\right\}
$$

6. Cubic correlation function:

$$
\psi_{j}\left(\theta ; d_{j}\right)=1-3 \xi_{j}^{2}+2 \xi_{j}^{3}, \quad \xi_{j}=\min \left\{1, \theta_{j}\left|d_{j}\right|\right\}
$$

7. Spline correlation function: $\psi_{j}\left(\theta ; d_{j}\right)= \begin{cases}1-5 \xi_{j}^{2}+30 \xi_{j}^{3}, & 0 \leq \xi_{j} \leq 0.2 \\ 1.25\left(1-\xi_{j}^{3}\right), & 0.2 \leq \xi_{j} \leq 1 \\ 0, & \xi_{j}>1\end{cases}$

where $\xi_{j}=\theta_{j}\left|d_{j}\right|$

For all the correlation functions described above, $d_{j}=x_{i}-x_{i}^{\prime}$. The hyperparameters associated with the covariance functions are determined either by using the maximum likelihood estimate (MLE) or by using the Bayes rule. A detailed account of MLE in the context of Kriging is given in [21].

\subsection{Polynomial Chaos Based Kriging (PC-Kriging)}

Finally, we discuss about a hybrid machine learning technique, referred to as the polynomial chaos based Kriging (PC-Kriging) [30-32]. PC-Kriging is a novel surrogate model that combine two well-known surrogates, namely, polynomial chaos expansion (PCE) [23, 25] and Kriging [19, 20]. PC-Kriging can be viewed as a Kriging model where the mean/trend function is modelled by using PCE. With this setup, it is possible to achieve a higher order accuracy as compared to PCE and Kriging.

PC-Kriging is a special kind of Kriging where the mean function of the Gaussian process is modelled by using polynomial chaos expansion. More specifically, $\boldsymbol{\mu}(\cdot)$ in Eq. (23) is represented by using Eq. 19. Under limiting condition, PC-Kriging converges either to PCE or to Kriging. Similar to Kriging, the hyperparameters in PC-Kriging are learned by maximizing the likelihood. For further details, interested readers may refer [19, 62].

Despite PC-Kriging's benefit over its individual PCE and Kriging, the hybrid metamodel suffers from the curse of dimensionality due to the factorial growth of unknown coefficients with a rise in the number of input parameters $N$. This limitation originates from the PCE component of PC-Kriging. To address this problem, a variant of PCKriging, referred to as Optimal PC- Kriging (OPC-Kriging) [31] was proposed. In OPC-Kriging, least angle regression (LAR) is used to only retain the important components of PCE. The OPC-Kriging follows an iterative algorithm where each polynomial can be added to the trend part one-by-one. Figure 4 presents a flowchart depicting the algorithm of OPC-Kriging. 


\section{Machine Learning Based Stochastic Impact Analysis}

A major objective of the present study is to determine stochastic response of low-velocity impact loading on composite plates following probabilistic and non-probabilistic frameworks. Both geometric and material uncertainties are considered in this work. To be specific, uncertainties in the composite plate stem from variation in the material properties, fibre orientation angle, twist angle, oblique impact angle, initial velocity of impactor, mass density of impactor, and thickness of target plates, inclusion of which in the analysis (following probabilistic and non-probabilistic approaches) is discussed here.

\subsection{Probabilistic Impact Analysis}

For probabilistic impact analysis, statistical descriptions of the stochastic inputs are necessary. To that end, the machine learning techniques discussed in previous section have been coupled with our in-house FE code for low-velocity impact analysis. For quantifying the uncertainty in the output responses, first input training samples are obtained using an appropriate design of experiment (sampling) scheme. Due to its simplicity and already proven superior performance, Sobol sequence $[63,64]$ has been used in this study. In the next step, the training outputs are obtained by using the actual FE solver. In the third step, the machine learning models are trained and the hyperparameters associated with the models are computed. Finally, Monte Carlo simulation is carried out based on the trained ML model to compute the probability density function of the output responses. A flowchart depicting the ML based probabilistic uncertainty quantification algorithm is presented in Fig. 5. For the current study, the following cases of uncertainties are considered at each lamina level (layer-wise uncertainty modelling)

1. Variation of fibre-orientation angle: $\psi_{1}\{\theta, E, G, v, \rho\}=\Theta[\{\theta(\tilde{\varsigma})\},\{E(\tilde{\varsigma})\},\{G(\tilde{\varsigma})\},\{v(\tilde{\varsigma})\},\{\rho(\tilde{\varsigma})\}]$

2. Variation of twist angle: $\psi_{2}\{\psi, \theta, E, G, v, \rho\}=\Theta[\psi,\{\theta(\tilde{\varsigma})\},\{E(\tilde{\zeta})\},\{G(\tilde{\zeta})\},\{v(\tilde{\zeta})\},\{\rho(\tilde{\zeta})\}]$

3. Variation of oblique impact angle: $\psi_{3}\{\beta, \theta, E, G, v, \rho\}=\Theta[\beta,\{\theta(\tilde{\varsigma})\},\{E(\tilde{\varsigma})\},\{G(\widetilde{\varsigma})\},\{v(\tilde{\varsigma})\},\{\rho(\tilde{\varsigma})\}]$

4. Variation of initial velocity of impactor: $\psi_{4}\{V, \theta, E, G, v, \rho\}=\Theta[V,\{\theta(\tilde{\varsigma})\},\{E(\tilde{\varsigma})\},\{G(\tilde{\varsigma})\},\{v(\tilde{\varsigma})\},\{\rho(\tilde{\varsigma})\}]$

5. Variation of mass density of impactor: $\psi_{5}\left\{\rho_{\text {imp }}, \theta, E, G, v, \rho\right\}=\Theta\left[\rho_{\text {imp }},\{\theta(\tilde{\varsigma})\},\{E(\tilde{\varsigma})\},\{G(\tilde{\varsigma})\},\{v(\tilde{\zeta})\},\{\rho(\tilde{\varsigma})\}\right]$

6. Variation of thickness of the plate: $\psi_{6}\left\{t_{p l t}, \theta, E, G, v, \rho\right\}=\Theta\left[t_{p l t},\{\theta(\tilde{\varsigma})\},\{E(\tilde{\varsigma})\},\{G(\tilde{\varsigma})\},\{v(\tilde{\varsigma})\},\{\rho(\tilde{\varsigma})\}\right]$

7. Variation in location of loading point:
Fig. 4 Flowchart for OPCKriging

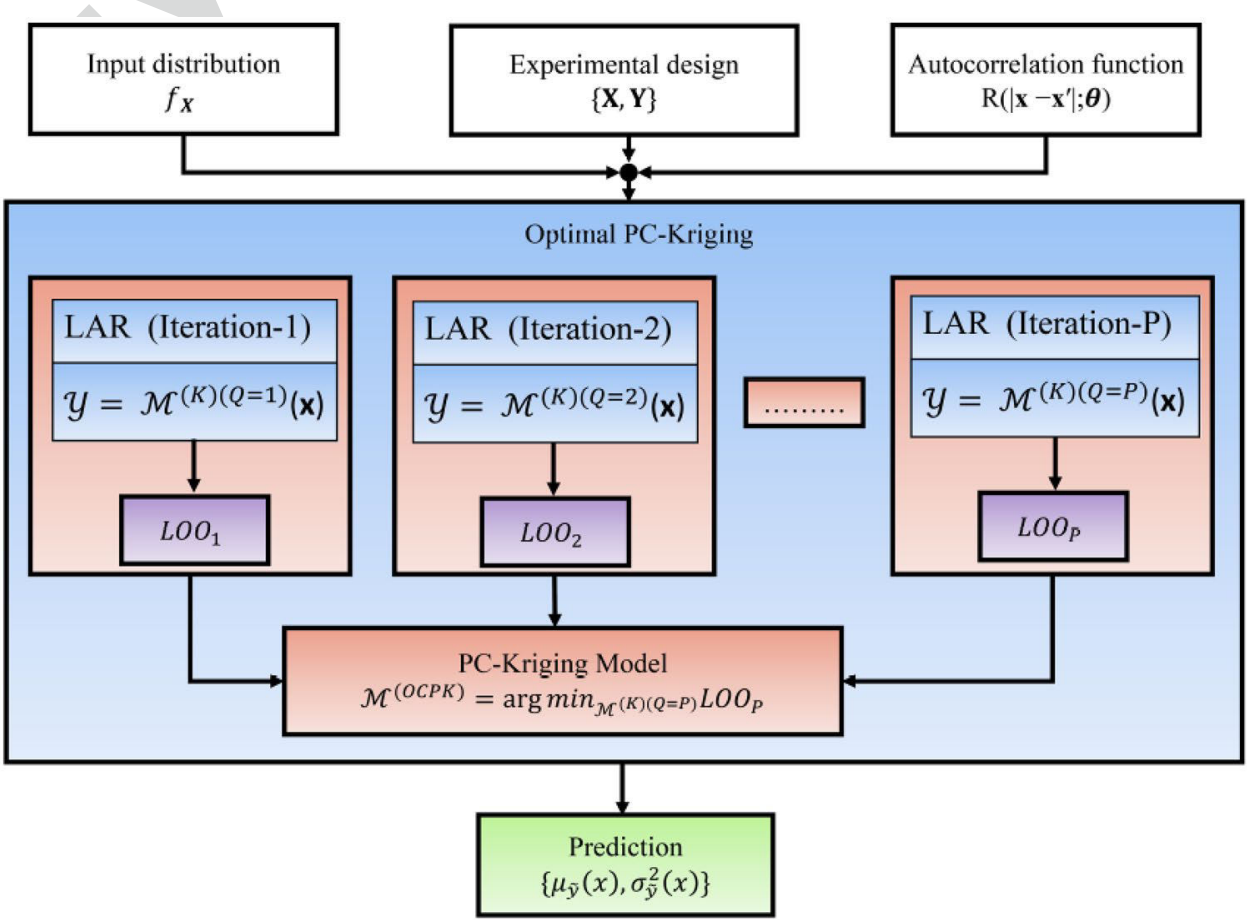




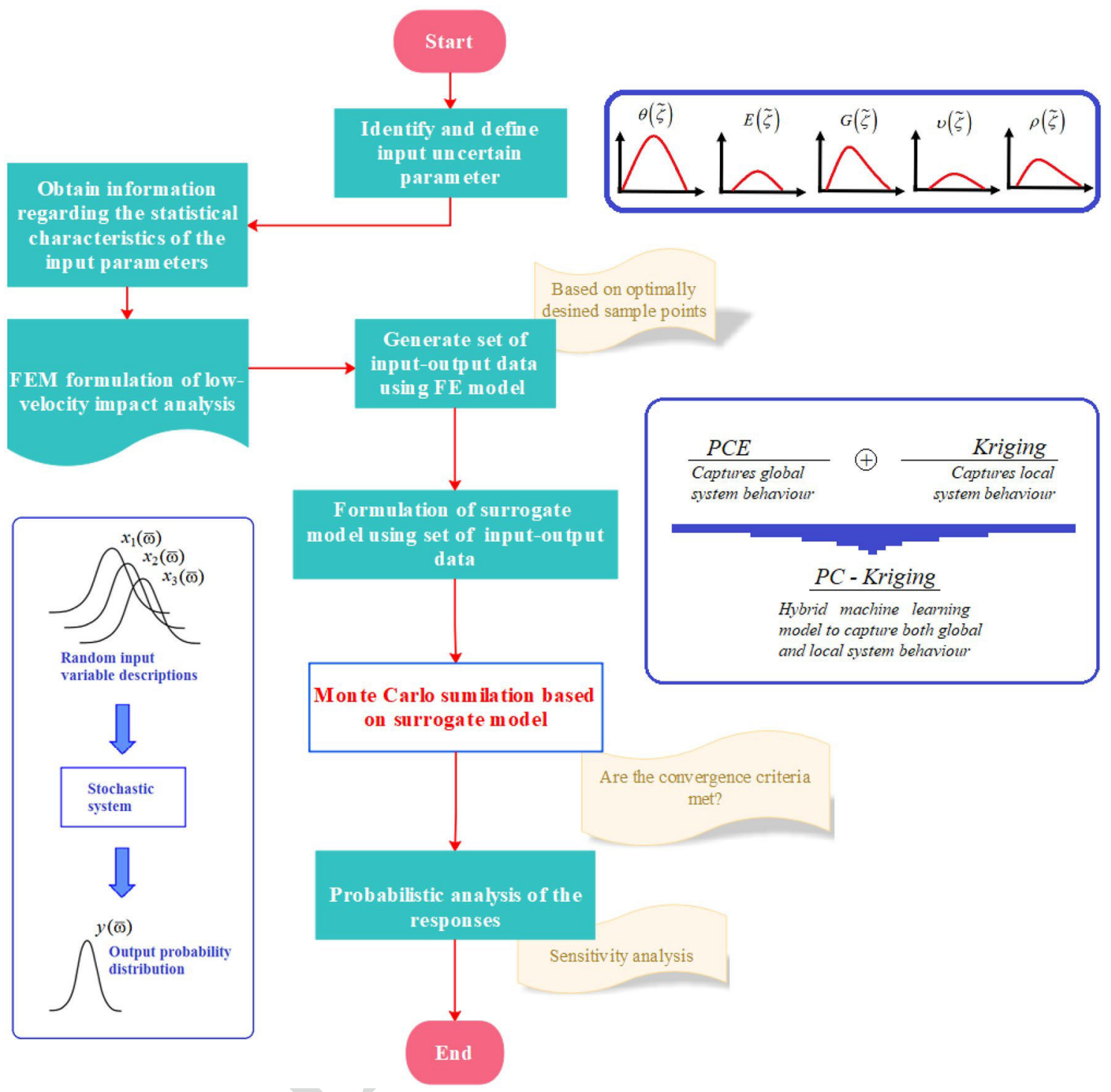

Fig. 5 Flowchart for probabilistic impact analysis based on hybrid machine learning models coupled with FE simulations

$\psi_{7}\left\{L_{p}, \theta, E, G, v, \rho\right\}=\Theta\left[L_{p},\{\theta(\tilde{\varsigma})\},\{E(\tilde{\varsigma})\},\{G(\tilde{\varsigma})\},\{v(\tilde{\varsigma})\},\{\rho(\tilde{\varsigma})\}\right]$

Here $\tilde{\zeta}$ is used to denote the stochastic representation of the system parameters. The parameters $E, G, v, \rho$ are the set of Young's moduli, shear moduli, mass density and Poisson's ratio in different directions, where the entire set of stochastic material properties is $\left\{E_{1}, E_{2}, E_{3}, G_{12}, G_{13}, G_{23}, v_{12}, v_{13}, v_{23}, v_{32}, v_{21}, v_{31}, \rho\right\}$. Unless otherwise mentioned, the degree of stochasticity from the respective deterministic values is taken as $\pm 10 \%$ (as per standard design practice) for each of the components in the set of material properties.

\subsection{Fuzzy Impact Analysis}

Although probabilistic analysis is more rigorous as it provides the probability distribution of the output responses, it is limited by the fact that we require probability distribution of the input variables for carrying out such analysis. In the real-life scenario, we may not have knowledge about the probability distribution of the input variables due to the requirement of extensive experimental characterization of the materials involving thousands of physical samples. Under such circumstances of sparse data availability, we have to opt for non- probabilistic analysis. Out of different non- probabilistic analysis methods available in literature, fuzzy based non-probabilistic analysis is employed 
for uncertainty quantification and propagation in lowvelocity impact analysis of the laminated composite plate. The fuzzy theory is employed in the intermediate stage between non-members and members known as membership function $\left[\mu_{p i}\right]$ that signifies the degree to which each component in the territory leads to the fuzzy set [65]. The triangular membership function is employed for the fuzzy number $\left[P_{i}\left(\tilde{\varsigma}_{\alpha}\right)\right]$ and expressed as

$P_{i}\left(\tilde{\varsigma}_{\alpha}\right)=\left[P_{i}^{U}, P_{i}^{M}, P_{i}^{L}\right]$

where $P_{i}^{M}, P_{i}^{U}, P_{i}^{L}$ denotes the mean value, upper bound and lower bound, respectively. Here $\tilde{\varsigma}_{\alpha}$ indicates the fuzzified variations corresponding to each $\alpha$-cut, where $\alpha$ is known as the degree of fuzziness or membership grade ranging from 0 to 1 . As an example, the Gaussian distribution can be approximated by using the triangle as shown in Fig. 6a, where the area under the Gaussian distribution is equal to the area under the triangular function [66]. The triangular fuzzy membership function is written as

$\mu_{P(i)}=\max \left[0,1-\frac{\left|X_{i}^{(j)}-X_{i}\right|}{\lambda}\right]$

where $\lambda=\left(2 \pi \sigma_{x}\right)^{1 / 2}, X_{i}$ and $\sigma_{x}$ represents the mean and standard deviation (S.D.) of the Gaussian distribution. In the present study, triangular membership function $\left[\mu_{P(i)}\right]$ is employed as

$\mu_{P(i)}=1-\left(P_{i}^{M}-P_{i}\right) /\left(P_{i}^{M}-P_{i}^{L}\right), \quad$ for $\left.\quad P_{i}^{L} \leq P_{i} \leq P_{i}^{M}\right)$ $\mu_{P(i)}=1-\left(P_{i}-P_{i}^{M}\right) /\left(P_{i}^{U}-P_{i}^{M}\right), \quad$ for $P_{i}^{M} \leq P_{i} \leq P_{i}^{U}$

$\mu_{P(i)}=0, \quad$ otherwise

By applying the $\alpha$-cut method, the fuzzy input number $P$ can be grouped into the set $\bar{P}_{i}$ of $(n+1)$ intervals $P_{i}^{(j)}$

$\bar{P}_{i}\left(\tilde{\zeta}_{\alpha}\right)=\left[P_{i}^{(0)}, P_{i}^{(1)}, P_{i}^{(2)}, P_{i}^{(3)}, \ldots, P_{i}^{(j)}, \ldots, P_{i}^{(n)}\right]$

where $n$ is the number of $\alpha$-cut levels. The interval of $j$-th level of $i$-th fuzzy number can be expressed

$P_{i}^{(j)}=\left[P_{i}^{(j, L)}, P_{i}^{(j, U)}\right]$

where $P_{i}^{(j, U)}$ and $P_{i}^{(j, L)}$ represent the upper and lower bound of the interval at the $j$-th level, respectively. At $j=n$, $P_{i}^{(n, U)}=P_{i}^{(n, L)}=P_{i}^{(n, M)}$. The superscript $U$ represents the upper bound, while $L$ denotes lower bound. The fuzzy input numbers are considered as the uncertain model parameters for the uncertainty analysis and an interval analysis is carried out at different $\alpha$-levels [67].

Even though in the present study we have considered triangular membership functions for the input parameters, the input membership functions can be augmented further depending on the availability of limited number of input dataset. In this work, we start by evaluating the deterministic solution at $\alpha=1$ level first and continue towards the lower $\alpha$ - cut levels using an interval analysis. As a special case, if the input-output relation of the problem in hand is monotonic in nature, computing the bounds of the fuzzy outputs becomes trivial. Unfortunately, for most real-life problems, the input-output relation is not monotonic in nature. Under such circumstances, a maximization and minimization algorithm involving multiple simulations is necessary. In this work, we proceed by first formulating the machine learning models as a surrogate to the actual FE code. Then we perform MCS on the trained machine learning models to compute the maximum and minimum values of the response quantities of interest for a particular $\alpha$-cut level. It is to be noted that only a single machine learning model is required in this case corresponding to $\alpha=0$ as the same model can be reused for other $\alpha$-cut levels. The number of actual FE simulations required in this study is therefore equal to the number of training samples needed to train the models of machine learning. The procedure of the present fuzzy impact approach is summarized in Figs. $6 \mathrm{~b}$ and 7.

\section{Numerical Investigation and Discussion}

In this work a glass-epoxy laminated composite plate having dimensions $L=1 \mathrm{~m}, b=1 \mathrm{~m}$ and $t=0.002 \mathrm{~m}$ is considered. Unless otherwise mentioned, the plate is considered to be subjected to normal and oblique impact loadings at the centre of the plate. The deterministic material properties of glass-epoxy are $E_{1}=38.6 \times 10^{9}$ $\mathrm{Pa}, \quad E_{2}=8.27 \times 10^{9} \mathrm{~Pa}, G_{12}=G_{13}=4.144 \times 10^{9} \mathrm{~Pa}$, $G_{23}=1.657 \times 10^{9} \mathrm{~Pa}, \rho=2600 \mathrm{~kg} / \mathrm{m}^{3}, v=0.26$ [68]. The diameter of spherical steel ball (impactor) is considered as $0.0127 \mathrm{~m}$. It is assumed that the fibre orientation angle may have a variation of $5 \%$ and the material properties may have a variation of $10 \%$ with respect to the deterministic values. Such variations are considered as per standard industrial practices; however, the current analysis can be extended to other percentages of variation, if required. Contact force (CF), impactor displacement (ID) and plate displacement (PD) are considered to be the output response variables. The in-house deterministic finite element code for impact analysis is validated with results of Sun and Chen [7] (refer to Fig. 8), wherein it is observed that the current results are extremely close to the results of literature. 


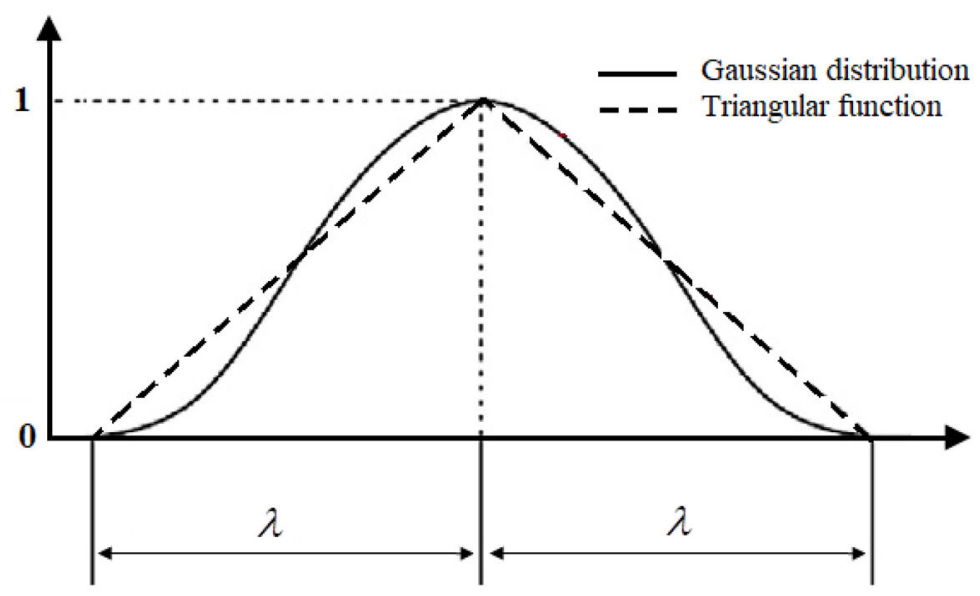

(a)

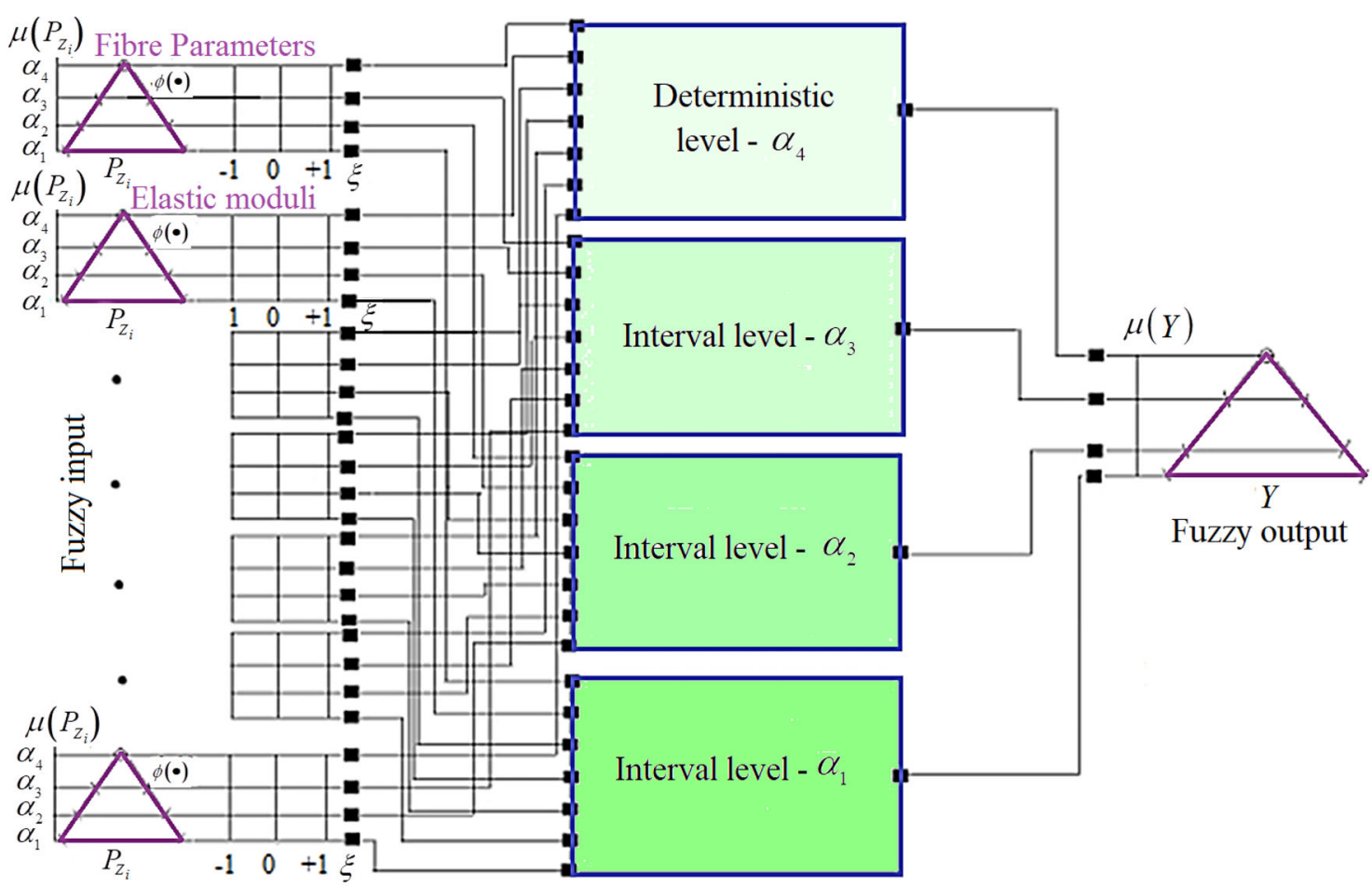

(b)

Fig. 6 a Triangular membership function approximated from Gaussian distribution. b Fuzzy analysis for different value of $\alpha$-cuts

\subsection{Deterministic Impact Analysis}

Deterministic numerical results of the low-velocity impact are discussed in this subsection (Tables 2, 3, 4, 5, 6, 7, 8) to study the basic and fundamental influence of different system parameters such as fibre-orientation angle, oblique impact angle, twist angle, initial velocity of impactor, mass density of impactor, thickness of plate and location of impact loading. Here we study four different crucial stacking sequences of the composite laminate: bending stiff laminate $\left(\left[0^{\circ} / 0^{\circ} / 30^{\circ} /-30^{\circ}\right] \mathrm{s}\right)$, cross ply laminate $\left(\left[90^{\circ} / 0^{\circ} / 90^{\circ} / 0^{\circ}\right] \mathrm{s}\right)$, torsion stiff laminate). The effects of stacking sequence on 


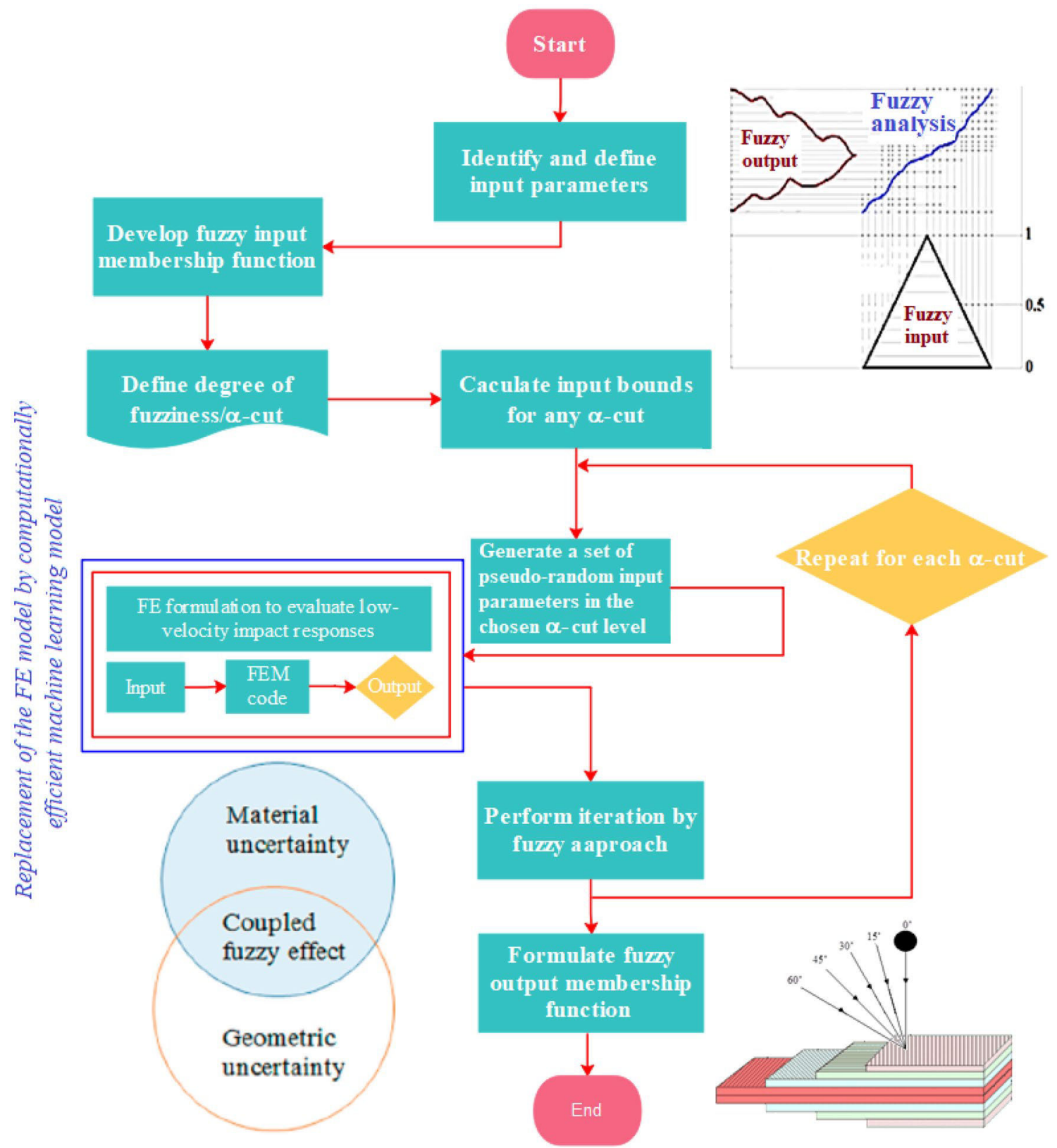

Fig. 7 Flowchart for non-probabilistic impact analysis based on fuzzy approach (Machine learning models are used instead of direct FE model, as indicated using a blue colour box)

low-velocity impact responses are furnished in Table 2. It is observed that the peak CF is highest for the torsion stiff laminates. On the other hand, peak ID and peak PD are found to be minimum for torsion stiff laminates and maximum for bending stiff laminates. Table 3 shows the variation of peak impact responses with the change in twist angle. The peak $\mathrm{CF}$ is found to increase with increase in twist angle. On the contrary, peak ID and peak PD decrease with the increase in twist angle. The influence of oblique impact angle on the responses is shown in Table 4. While peak CF and peak PD 


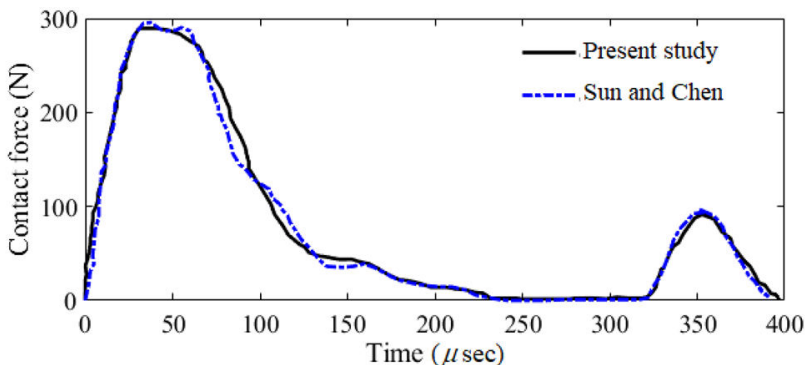

(a)

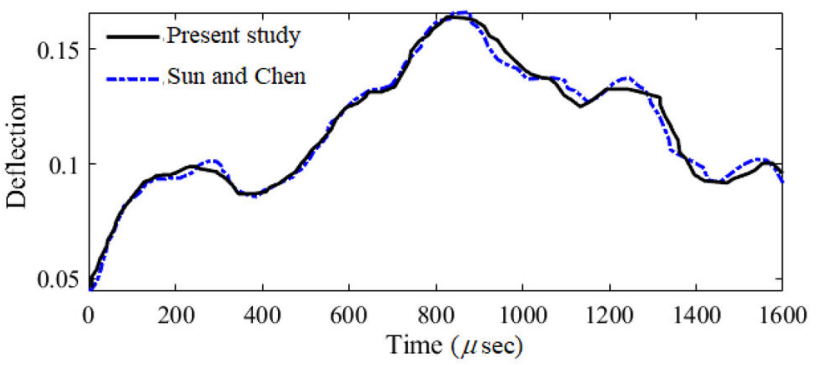

(b)

Fig. 8 Time histories of $\mathbf{a}$ contact force and $\mathbf{b}$ deflection of glass epoxy composite plates considering a centrally impacted bending stiff laminated composite plate $\left( \pm 0^{\circ} / \pm 30^{\circ}\right)$ with dimension $L=1 \mathrm{~m}$, $b=1 \mathrm{~m}$, and $t=0.002 \mathrm{~m}, \psi=0^{\circ}, \beta=0^{\circ}$, initial velocity of impactor $=5 \mathrm{~m} / \mathrm{s}$, diameter of spherical steel ball $=0.0127 \mathrm{~m}$, mass density of impactor $(\rho)=0.0085 \frac{N-s}{\mathrm{~cm}^{4}}[7]$

Table 2 Effect of stacking sequence (quasi-isotropic stiff, torsion stiff, cross ply and bending stiff laminates on low-velocity impact responses considering $t=0.002 \mathrm{~m}, \psi=0^{\circ}, \beta=0^{\circ}, \mathrm{V}=5 \mathrm{~m} / \mathrm{s}$, $\rho=0.0085 \frac{\mathrm{N}-s}{\mathrm{~cm}^{4}}$

\begin{tabular}{llll}
\hline Stacking sequence & \multicolumn{3}{l}{ Impact responses (maximum value) } \\
\cline { 2 - 4 } & CF $(\mathrm{N})$ & ID $(\mathrm{m})$ & PD $(\mathrm{m})$ \\
\hline Bending stiff & 744.7855 & 0.000225 & 0.090134 \\
Quasi-isotropic stiff & 770.0546 & 0.000221 & 0.0854 \\
Cross ply & 770.45 & 0.000219 & 0.08794 \\
Torsion stiff & 773.31 & 0.000219 & 0.08548 \\
\hline
\end{tabular}

Table 3 Effect of twist angle $(\psi)$ on low-velocity impact responses with considering $t=0.002 \mathrm{~m}, \beta=0^{\circ}, V=5 \mathrm{~m} / \mathrm{s}, \rho=0.0085 \frac{N-s}{\mathrm{~cm}^{4}}$, bend-

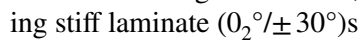

\begin{tabular}{llll}
\hline Twist angle & \multicolumn{3}{l}{ Impact responses (maximum value) } \\
\cline { 2 - 4 } & CF (N) & ID (m) & PD (m) \\
\hline$\psi=0^{\circ}$ & 744.7855 & 0.000227 & 0.090134 \\
$\psi=15^{\circ}$ & 776.3958 & 0.000221 & 0.0882 \\
$\psi=30^{\circ}$ & 874.1484 & 0.000206 & 0.0833 \\
$\psi=45^{\circ}$ & 1053.9 & 0.000188 & 0.07413 \\
\hline
\end{tabular}

Table 4 Effect of oblique impact angle $(\beta)$ on low-velocity impact responses with considering $t=0.002 \mathrm{~m}, \psi=0^{\circ}, V=5 \mathrm{~m} / \mathrm{s}, \rho=0.0085$ $\frac{N-s}{\mathrm{~cm}^{4}}$, bending stiff laminate $\left(0_{2}{ }^{\circ} \pm 30^{\circ}\right) \mathrm{s}$

\begin{tabular}{llll}
\hline $\begin{array}{l}\text { Oblique impact } \\
\text { angle }\end{array}$ & \multicolumn{3}{l}{ Impact responses (maximum value) } \\
\cline { 2 - 4 } & $\mathrm{CF}(\mathrm{N})$ & ID $(\mathrm{m})$ & $\mathrm{PD}(\mathrm{m})$ \\
\hline$\beta=0^{\circ}$ & 744.7855 & 0.000225 & 0.090134 \\
$\beta=15^{\circ}$ & 724.1631 & 0.000232 & 0.08965 \\
$\beta=30^{\circ}$ & 661.4398 & 0.000251 & 0.087791 \\
$\beta=45^{\circ}$ & 553.4121 & 0.00029 & 0.084967 \\
\hline
\end{tabular}

Table 5 Effect of initial velocity of impactor on low-velocity impact responses with considering $t=0.002 \mathrm{~m}, \psi=0^{\circ}, \beta=0^{\circ}, \rho=0.0085 \frac{\mathrm{N-s}}{\mathrm{cm}^{4}}$, bending stiff laminate $\left(0_{2} \% \pm 30^{\circ}\right) \mathrm{s}$

\begin{tabular}{llll}
\hline $\begin{array}{l}\text { Initial velocity of } \\
\text { impactor }(\mathrm{m} / \mathrm{s})\end{array}$ & \multicolumn{3}{l}{ Impact responses (maximum value) } \\
\cline { 2 - 4 } & $\mathrm{CF}(\mathrm{N})$ & $\mathrm{ID}(\mathrm{m})$ & $\mathrm{PD}(\mathrm{m})$ \\
\hline $\mathrm{V}=5$ & 744.7855 & 0.000227 & 0.090134 \\
$\mathrm{~V}=10$ & 1549.402 & 0.00042 & 0.177863 \\
$\mathrm{~V}=15$ & 2365.073 & 0.000606 & 0.263738 \\
$\mathrm{~V}=20$ & 3193.182 & 0.000789 & 0.349809 \\
\hline
\end{tabular}

Table 6 Effect of mass density of impactor $\left(\rho\right.$ in $\frac{N-s}{\mathrm{~cm}^{4}}$ ) on low-velocity impact responses with considering $t=0.002 \mathrm{~m}, \psi=0^{\circ}, \beta=0^{\circ}$, $V=5 \mathrm{~m} / \mathrm{s}$, bending stiff laminate $\left(0_{2}{ }^{\circ} / \pm 30^{\circ}\right) \mathrm{s}$

\begin{tabular}{llll}
\hline $\begin{array}{l}\text { Mass density of } \\
\text { impactor }\end{array}$ & \multicolumn{4}{l}{ Impact responses (maximum value) } \\
\cline { 2 - 4 } & CF (N) & ID (m) & PD (m) \\
\hline$\rho=75 \times 10^{-4}$ & 719.9314 & 0.00021 & 0.08149 \\
$\rho=80 \times 10^{-4}$ & 733.6016 & 0.000219 & 0.085852 \\
$\rho=85 \times 10^{-4}$ & 744.7855 & 0.000227 & 0.090134 \\
$\rho=90 \times 10^{-4}$ & 755.3778 & 0.000235 & 0.094109 \\
$\rho=95 \times 10^{-4}$ & 766.9816 & 0.000242 & 0.098161 \\
\hline
\end{tabular}

peak $\mathrm{CF}, \mathrm{PD}$ and ID are maximum at point 2 , point 3 and point 3 , respectively. 
Table 7 Effect of thickness of plate $(t)$ on low-velocity impact responses with considering $\psi=0^{\circ}, \beta=0^{\circ}, V=5 \mathrm{~m} / \mathrm{s}, \rho=0.0085 \frac{\mathrm{N-s}}{\mathrm{cm}^{4}}$, bending stiff laminate $\left(0_{2}^{\circ} \% 30^{\circ}\right) \mathrm{s}$

\begin{tabular}{llll}
\hline $\begin{array}{l}\text { Thickness of plate } \\
(\mathrm{m})\end{array}$ & \multicolumn{4}{l}{ Impact responses (maximum value) } \\
\cline { 2 - 4 } & $\mathrm{CF}(\mathrm{N})$ & ID $(\mathrm{m})$ & PD $(\mathrm{m})$ \\
\hline$t=0.002$ & 322.9597 & 0.000548 & 0.266644 \\
$t=0.004$ & 744.7855 & 0.000225 & 0.090134 \\
$t=0.006$ & 1054.777 & 0.000188 & 0.050085 \\
$t=0.008$ & 1248.632 & 0.000176 & 0.033335 \\
\hline
\end{tabular}

Table 8 Effect of location of impactor contacting point on lowvelocity impact responses with dimension $t=0.002 \mathrm{~m}, \psi=0^{\circ}, \beta=0^{\circ}$, $V=5 \mathrm{~m} / \mathrm{s}, \rho=0.0085 \frac{N-s}{\mathrm{~cm}^{4}}$, bending stiff laminate $\left(0_{2}^{\circ} / \pm 30^{\circ}\right) \mathrm{s}$ (location of impact points on the laminated composite plate is indicated in the inset of Fig. 19a)

\begin{tabular}{llll}
\hline Location of impactor & \multicolumn{3}{l}{ Impact responses (maximum value) } \\
\cline { 2 - 4 } & CF $(\mathrm{N})$ & ID $(\mathrm{m})$ & PD $(\mathrm{m})$ \\
\hline Location 1 & 731.8873 & 0.000228 & 0.075777 \\
Location 2 & 744.7855 & 0.000225 & 0.090134 \\
Location 3 & 735.177 & 0.000229 & 0.124411 \\
\hline
\end{tabular}
preceding section, Sobol sequence is adopted in this study to generate samples for training ML model. However, the optimal number of training samples required still needs to be determined. To that end, a study by varying the number of training samples has been carried out. Figure 9 shows the PDF of responses (for direct MCS and PCE-Kriging based MCS) with respect to training sample size of 32, 64, 128, 256, 512 and 1024. For all the three output responses, the results obtained using 512 training samples are almost identical to those obtained using 1024 samples. Based on this observation, we conclude that 512 is the optimal number of training samples. Note that all the subsequent results are obtained by training the surrogate with 512 training samples.

5.2.1.2 PCE Versus Kriging Versus PC-Kriging: A Comparative Study The surrogate PC-Kriging is developed by combine PCE and Kriging. In this section, we examine the performance of the three surrogate models (PCE, Kriging and PC-Kriging) in the context of probabilistic low-velocity impact analysis. To that end, coefficient of determination $\left(R^{2}\right)$ and root mean square error (RMSE) have been computed corresponding to training sample size of $32,64,128$, 256,512 and 1024 . Figure 10 shows the $R^{2}$ and RMSE corresponding to the different training sample size and the three surrogate models.

It is observed that PC-Kriging consistently outperforms PCE and Kriging; although the results obtained using PCE are found to be extremely close to the PC-Kriging results. Moreover, similar to the observations in previous section, the results obtained corresponding to sample size of 512 and 1024 are almost identical (with $R^{2}$ close to 1 ), indicating that the surrogate models converge at 512 training samples. Figure 11 shows the probability density functions obtained using PCE, Kriging and PC-Kriging, wherein the results are compared with benchmark Monte Carlo simulation results. For all the three cases, PC-Kriging is found to yield best results followed by PCE, establishing the superiority of PC-Kriging over PCE and Kriging. All the subsequent results in this paper are obtained using PC-Kriging trained with 512 training samples. It can be noted in this context that stochastic analysis of composite structures leading to the uncertainty quantification of different global responses have recently received significant attention from the scientific community [69-79]. However, most of these studies consider a single machine learning algorithm to map the stochastic input-output domain. The current investigation is the first attempt to investigate the performance of hybrid machine learning algorithms for any structural response of composite structures. 


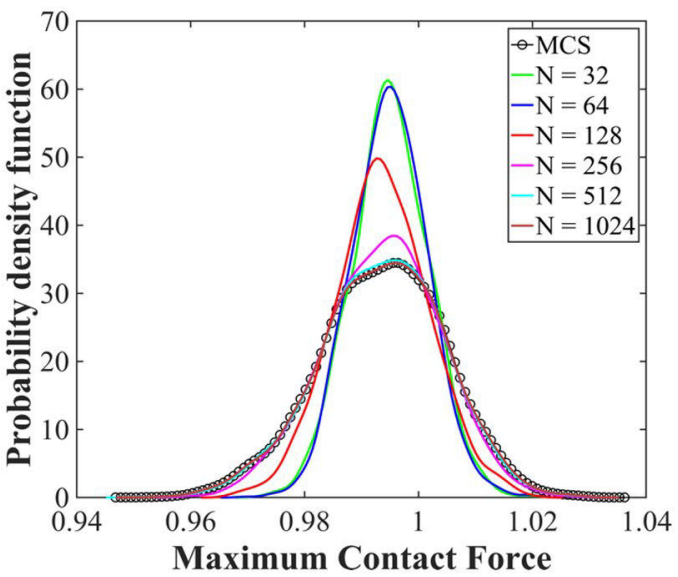

(a)

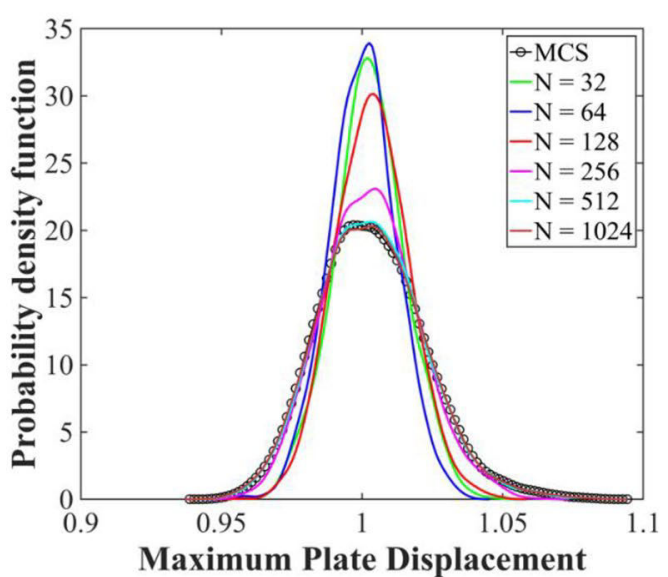

(b)

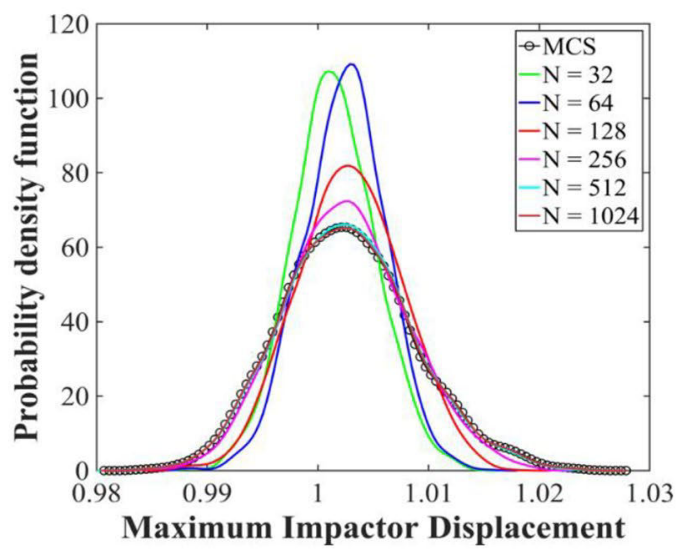

(c)

Fig. 9 Convergence study for PC-Kriging with respect to the number of training samples. For all the three responses, PC-Kriging converges at 512 training samples

\subsubsection{Probabilistic Impact Analysis}

Having established the superiority of PC-Kriging over PCE and Kriging, we present results for probabilistic impact analysis in this subsection based on the PC-Kriging assisted approach. The results presented here correspond to the impact location at the centre of the plate, unless otherwise mentioned. Figures 12 shows the variation of contact force, displacements of impactor and plate, and velocity of impactor with respect to time history for different stacking sequences. The figure also shows the corresponding stochastic response bounds arising due to the source- uncertainties. It is found that contact force initially increases at a significant rate with time and then decreases up to zero gradually. Impactor and plate displacements are noticed to gradually increase to a peak value and then reduce with the elapse of time. The velocity of the impactor reduces gradually over time and becomes constant after a certain duration.

The influence of fibre orientation angle in composite laminates is shown in Fig. 13. It is observed that the peak $\mathrm{CF}$ occurs for the torsion stiff laminates. The effects of twist angle on the critical impact responses are furnished in Fig. 14. In this case, the CF increases with the increase in twist angle, while peak ID and peak PD have a reverse trend. In case of impact loading, impact angle has a significant effect on the critical impact responses as shown in Fig. 15. The peak CF and peak PD decreases with the increase in impact angle from $0^{\circ}$ to $45^{\circ}$ while peak PD is found to have a reverse trend. All the impact responses increase with the increase in the initial velocity of the impactor as shown in 
Fig. 10 PCE vs Kriging versus PC-Kriging (PC-Kriging is found to yield the best results)

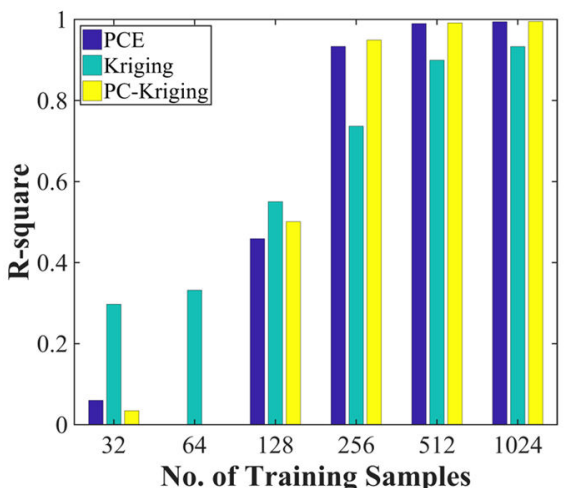

(a) Maximum Contact Force (r-square)

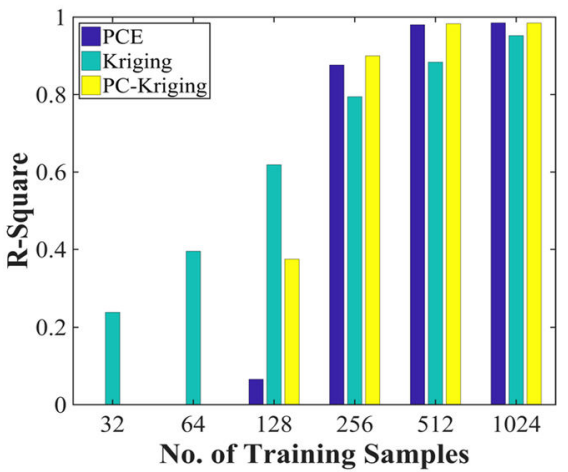

(c) Maximum plate displacement (r-square)

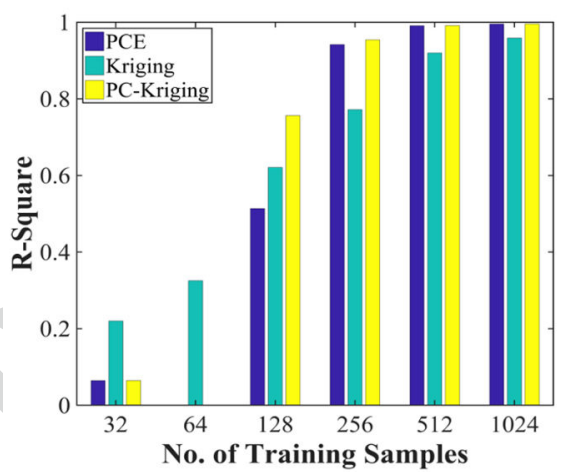

(e) Maximum impactor displacement (r- square)

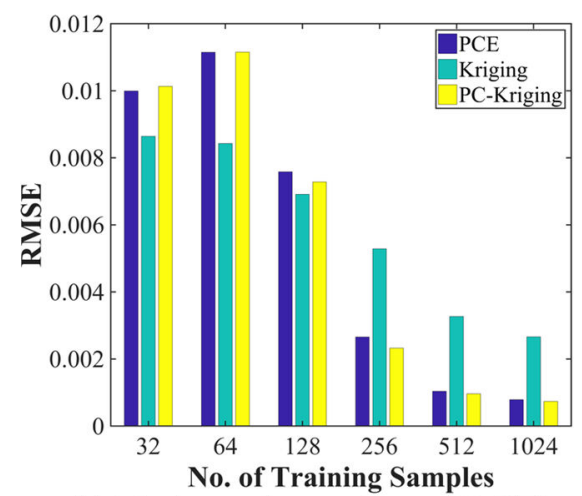

(b) Maximum Contact Force (RMSE)

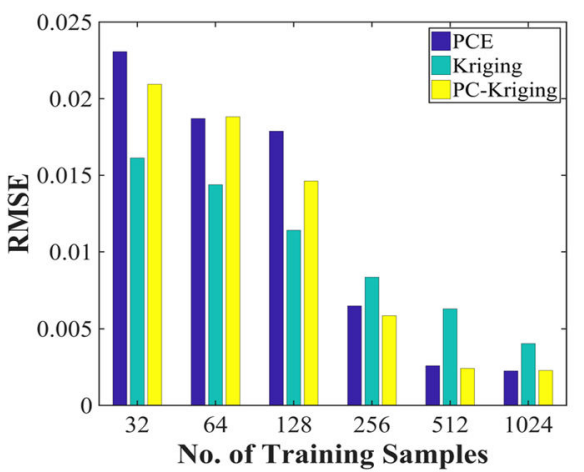

(d) Maximum plate displacement (RMSE)

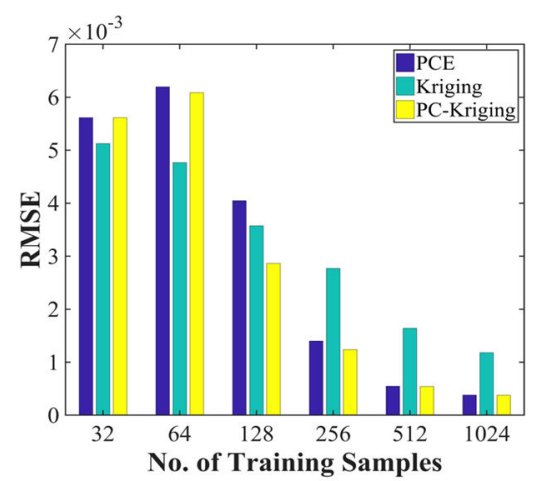

(f) Maximum impactor displacement (RMSE)
Fig. 16 due to the increase in kinetic energy. The standard deviation of the response parameters is also found to follow a similar trend for initial velocity of impactor. The increase in impactor mass density also leads to an increase of all impact responses for the same reason as shown in Fig. 17. The effect of plate thickness on the impact responses are shown in Fig. 18, wherein contact force is found to increase with the increase in plate thickness. On the other hand, the displacement of the impactor and plate displacement reduce as the plate thickness increases. The standard deviation of the response parameters is also found to follow a similar trend for thickness. The effect of location of impactor 


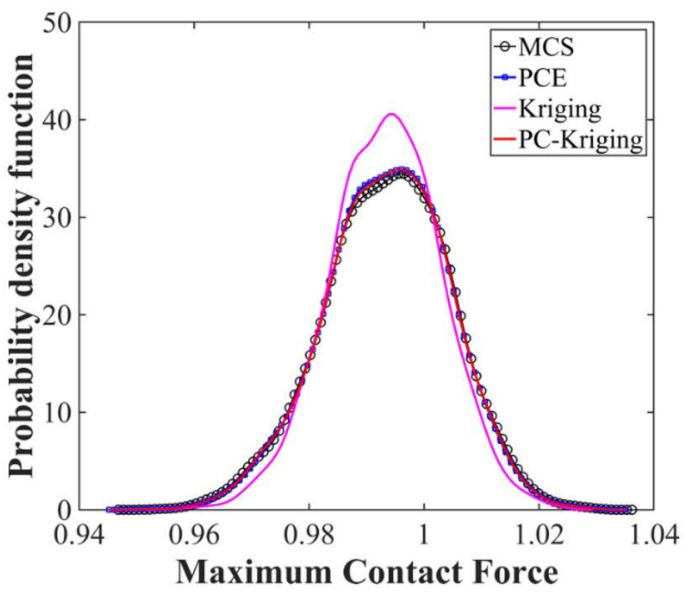

(a)

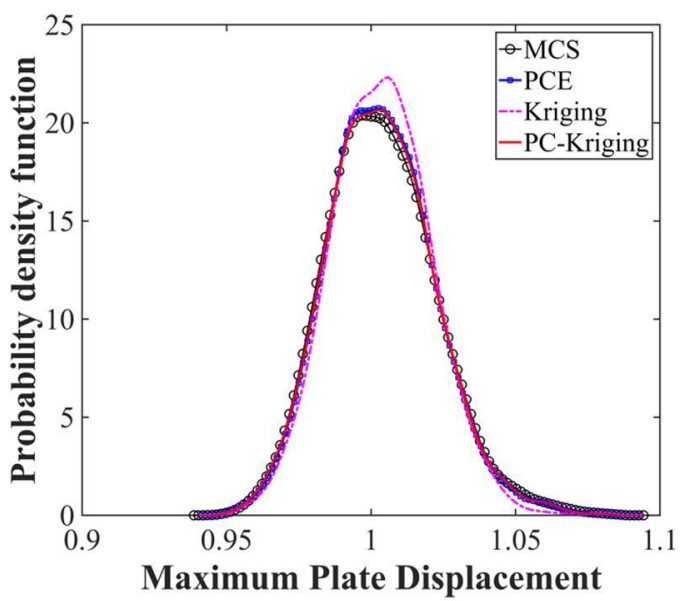

(b)

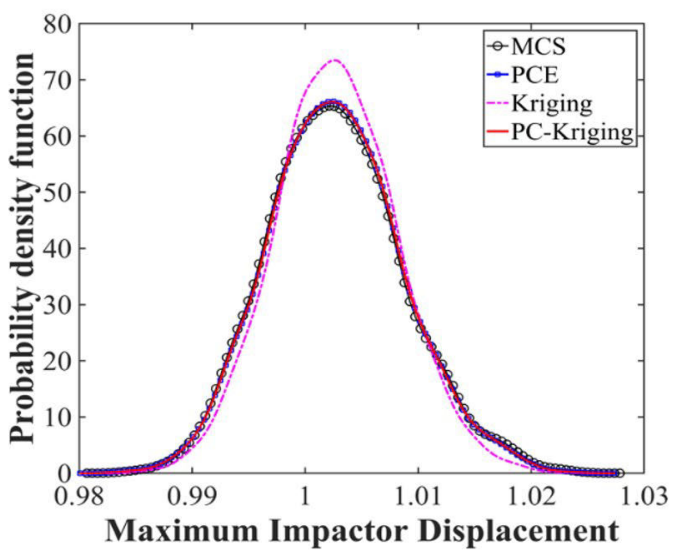

(c)

Fig. 11 Comparison of PCE, Kriging and PC-Kriging results. All the three models are trained with 512 training samples

contacting point on the impact responses is shown in Fig. 19. It is observed that contact force is maximum at the location 2 i.e. centre of the plate, while plate displacement and impactor displacement are maximum at location 3 . The relative coefficient of variation is shown for various influencing system parameters in Fig. 20 to understand about their rela- 80 . tive degree of influence on the impact response parameters. $\quad 80$ : The coefficient of variation (COV) is obtained by taking the 80 ratio of standard deviation to mean of the responses. Here 80 the relative coefficient of variation (RCOV) is computed by 80 : 


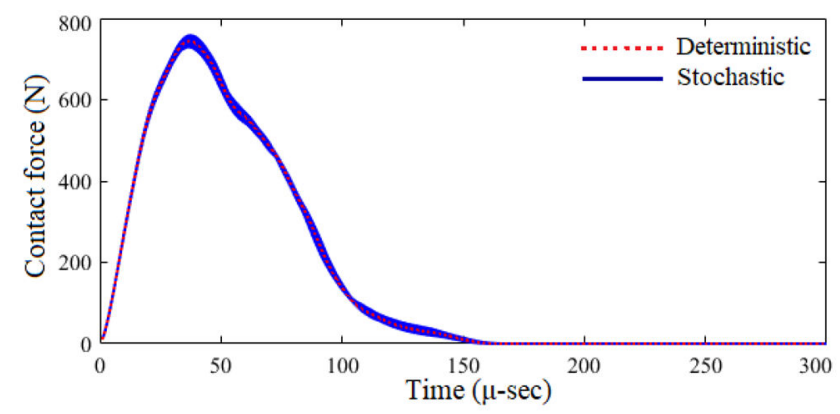

(a)

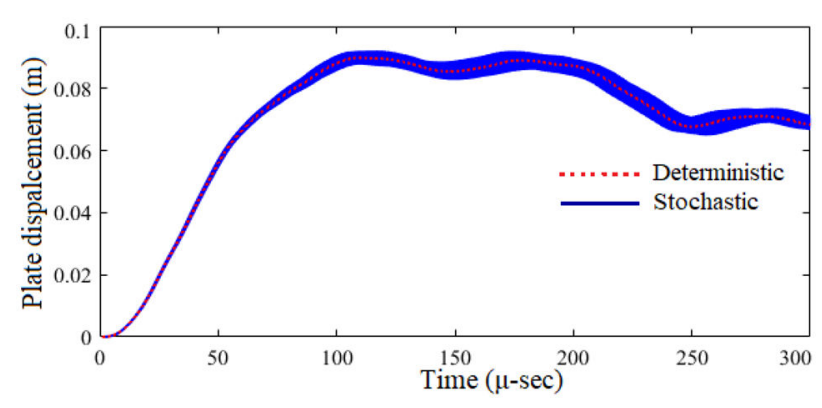

(b)

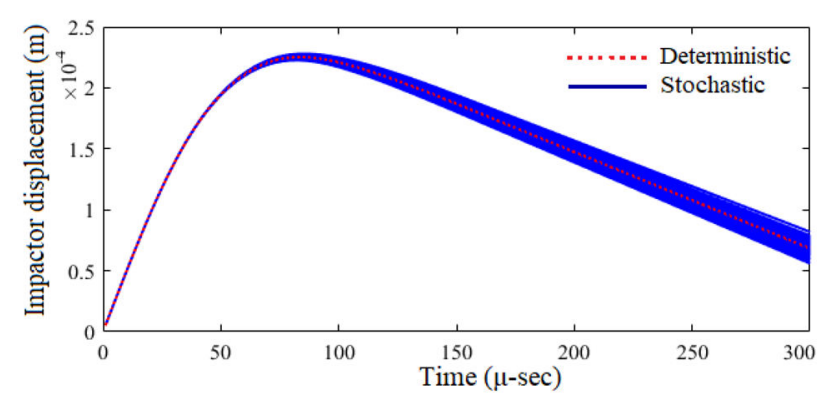

(c)

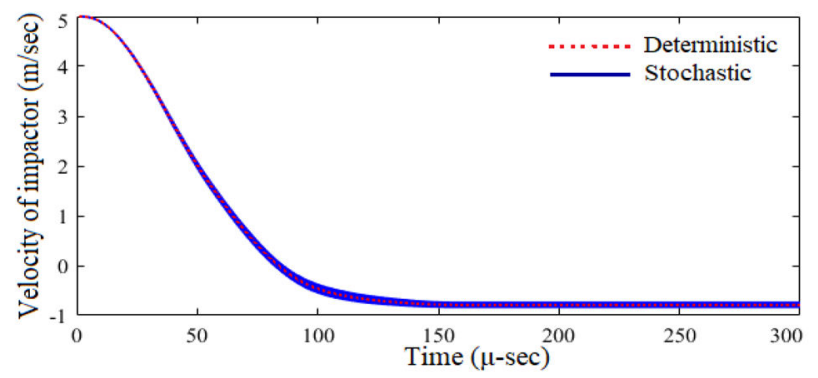

(d)

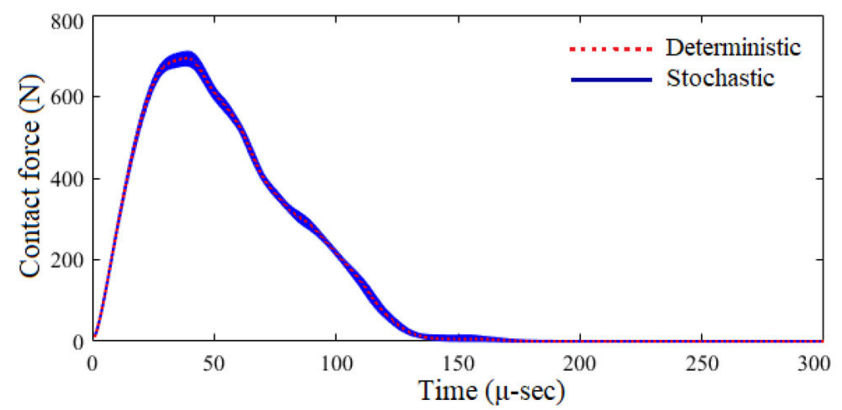

(e)

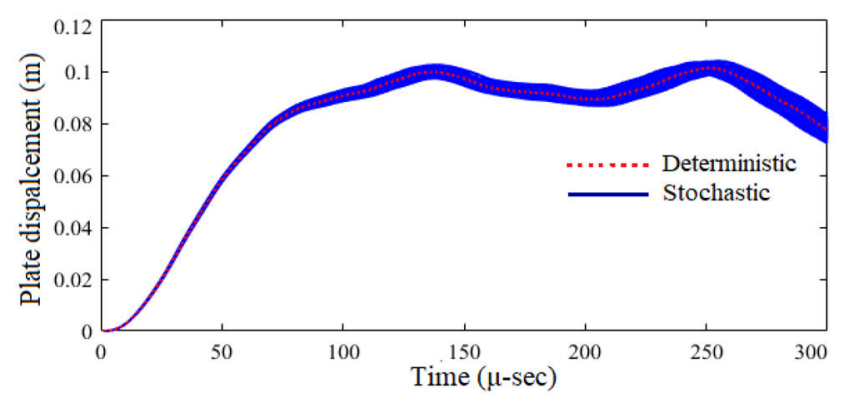

(f)

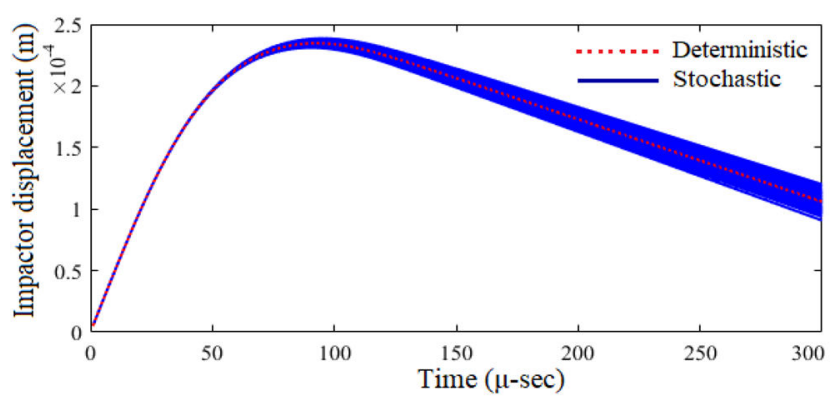

(g)

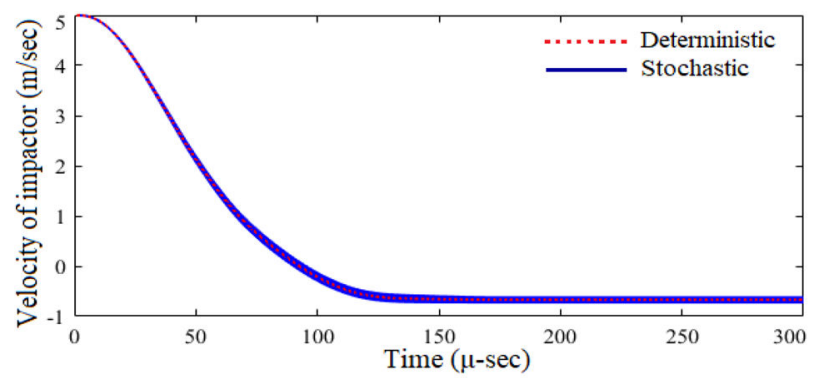

(h) 


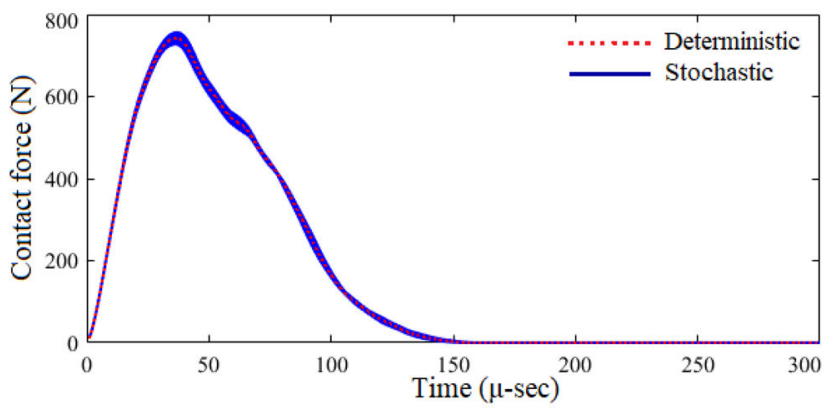

(i)

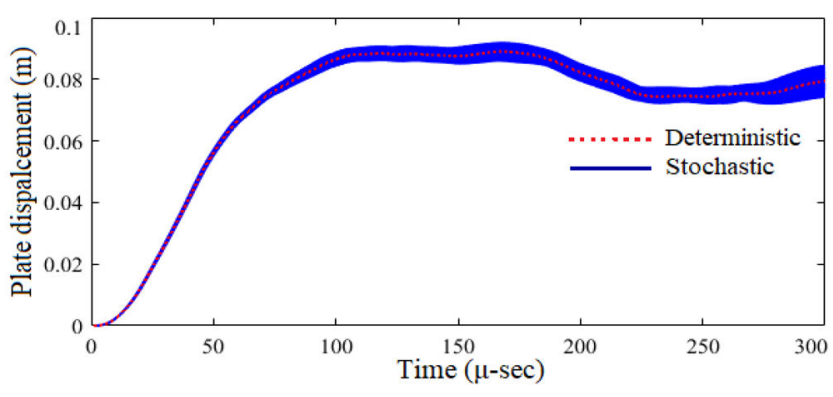

(j)

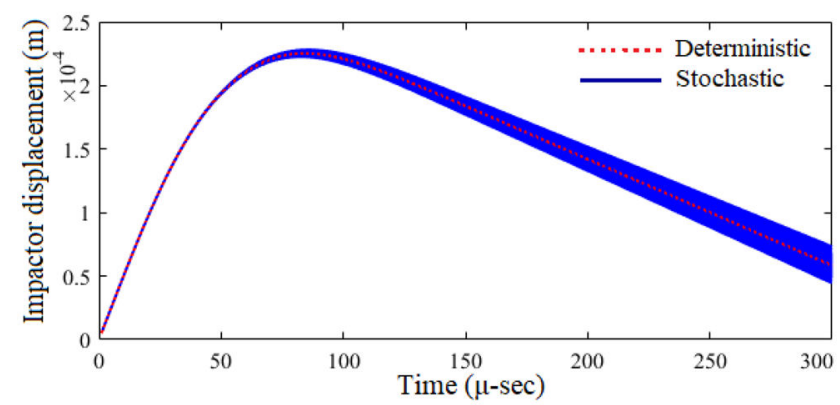

(k)

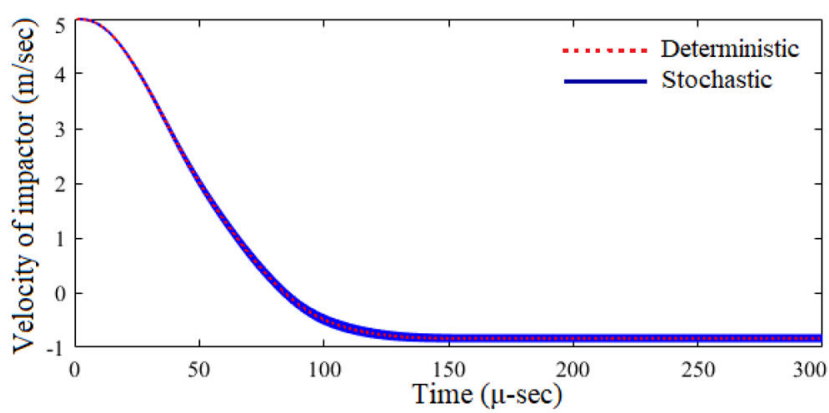

(1)

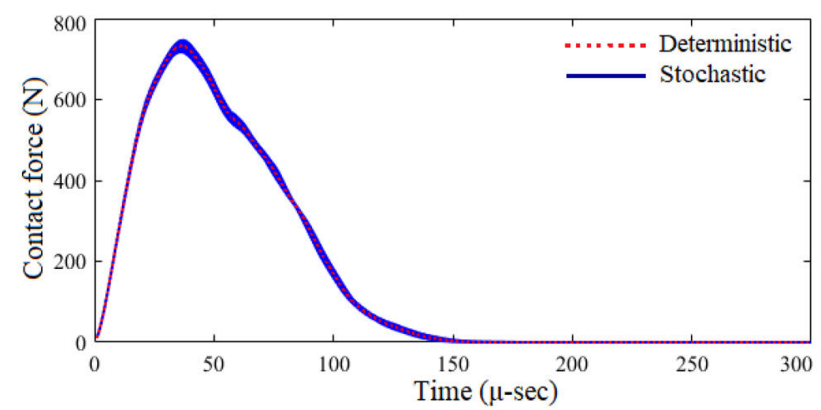

(m)

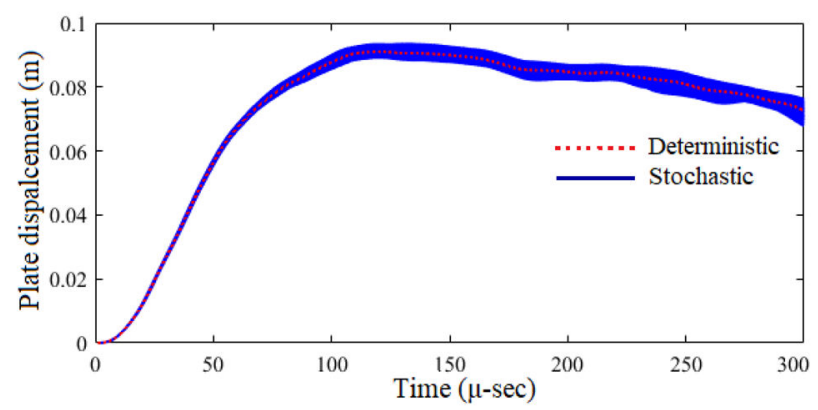

(n)

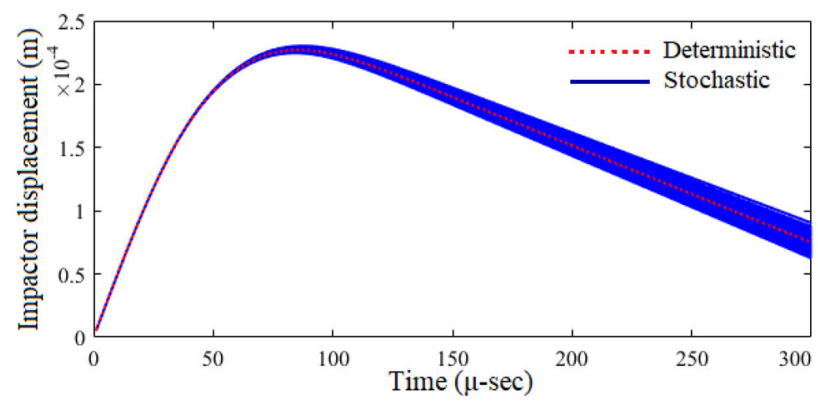

(o)

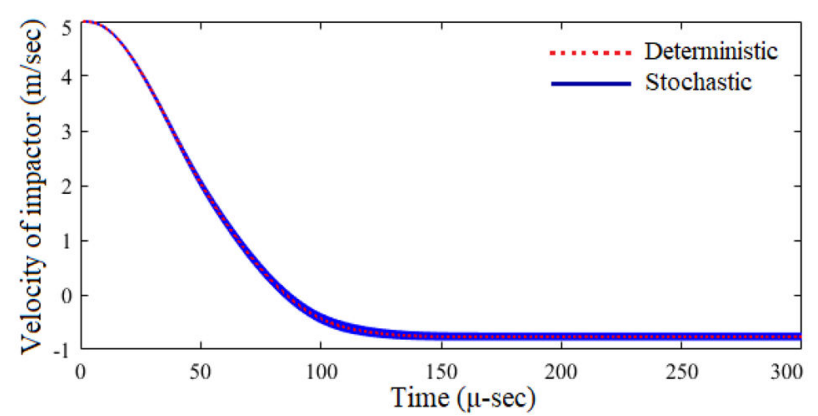

(p)

Fig. 12 Stochastic variation of the time history of low-velocity impact responses for different stacking sequences of the composite plate a-d for torsion stiff laminate $\left(45^{\circ},-45^{\circ}, 45^{\circ},-45^{\circ}\right) \mathrm{s}$, e-h for bending stiff laminate $\left(0^{\circ}, 0^{\circ}, 30^{\circ},-30^{\circ}\right) \mathrm{s}$ considering $t=0.002 \mathrm{~m}, \psi=0^{\circ}, \beta=0^{\circ}$, $V=5 \mathrm{~m} / \mathrm{s}, \rho=0 \cap n \cap 5 \frac{N-s}{\mathrm{~cm}^{4}}$, and $\Delta t=1$ micro-second. Stochast $\quad$ iation of the time history of low-velocity impact responses for different stacking sequences $\varepsilon-\bar{d}^{-}$)r cross ply laminate $\left(90^{\circ}, 0^{\circ}, 90^{\circ}, 0^{\circ}\right) \mathrm{s}$, or quasi-Isotropic stiff laminate $\left(0^{\circ}, 45^{\circ},-45^{\circ}, 90^{\circ}\right) \mathrm{s} \mathrm{considering} t=0.002 \mathrm{~m}$, $\psi=0^{\circ}, \beta=0^{\circ}, V=5 \mathrm{~m} / \mathrm{s}, \rho=0.0085 \frac{N-s}{\mathrm{~cm}^{4}}$, and $\Delta t=1$ micro-second 


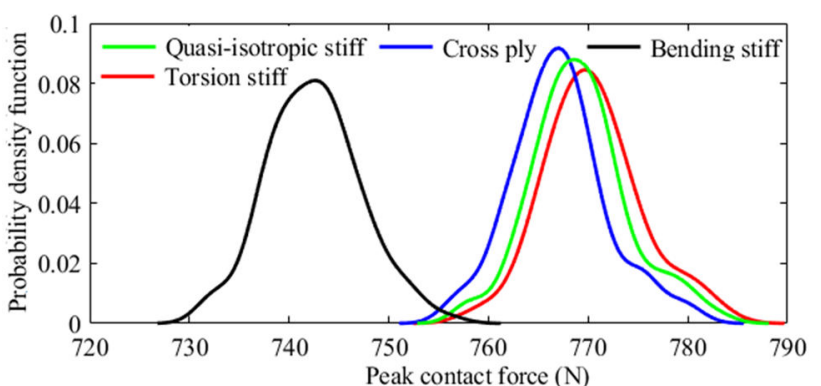

(a)

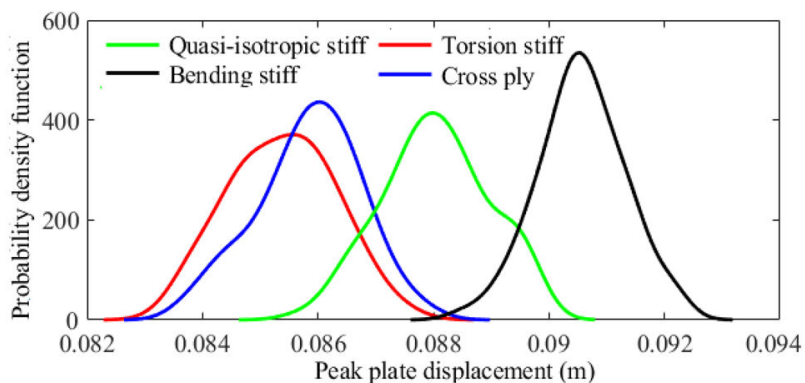

(b)

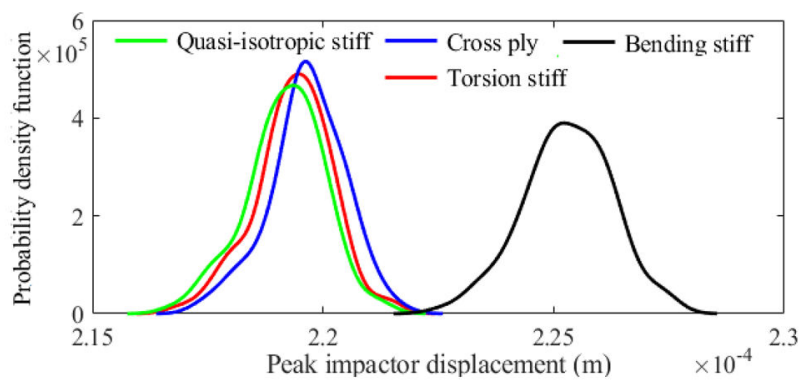

(c)

Fig. 13 Effect of variation of stacking sequence (quasi-isotropic stiff laminate $\left(0^{\circ}, 45^{\circ},-45^{\circ}, 90^{\circ}\right) \mathrm{s}$, torsion stiff laminate $\left(45^{\circ},-45^{\circ}, 45^{\circ}\right.$, $\left.-45^{\circ}\right) \mathrm{s}$, cross ply laminate $\left(90^{\circ}, 0^{\circ}, 90^{\circ}, 0^{\circ}\right) \mathrm{s}$ and bending stiff laminate $\left(0^{\circ}, 0^{\circ}, 30^{\circ},-30^{\circ}\right) \mathrm{s}$ on low-velocity impact responses considering $t=0.002 \mathrm{~m}, \psi=0^{\circ}, \beta=0^{\circ}, V=5 \mathrm{~m} / \mathrm{s}, \rho=0.0085 \frac{N-s}{\mathrm{~cm}^{4}}$

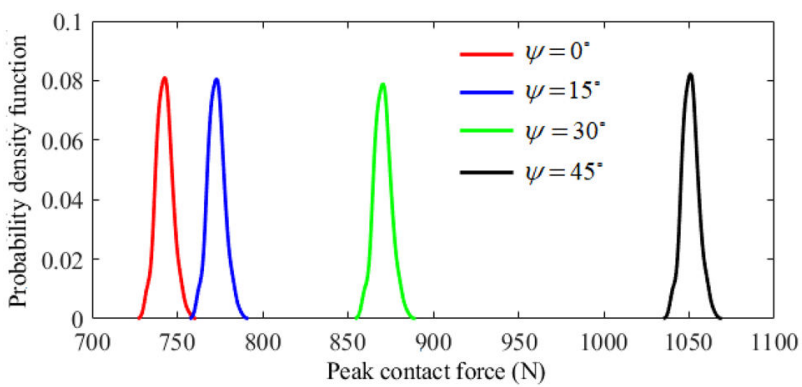

(a)

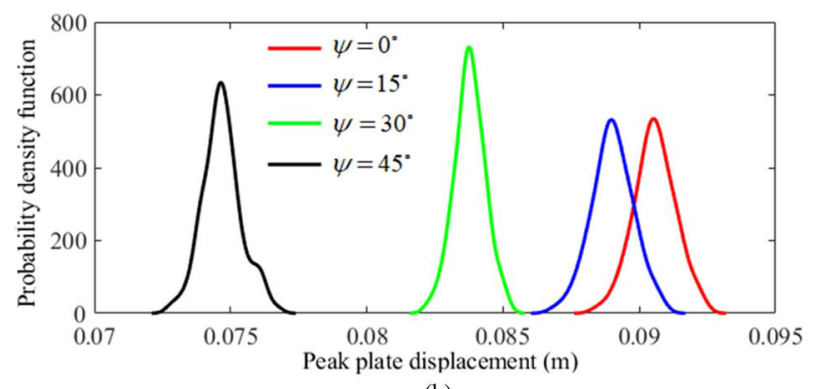

(b)

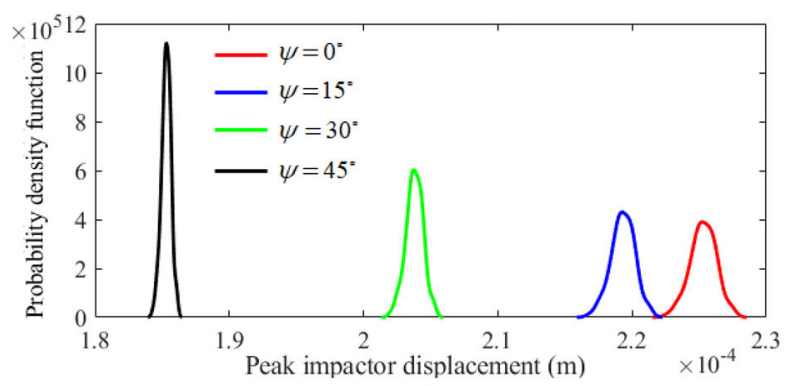

(c)

Fig. 14 Effect of variation of twist angle $(\psi)$ on PDF plots of lowvelocity impact responses considering $t=0.002 \mathrm{~m}, \beta=0^{\circ}, V=5 \mathrm{~m} / \mathrm{s}$, $\rho=0.0085 \frac{N-s}{\mathrm{~cm}^{4}}$, bending stiff laminate $\left(0_{2} \% \pm 30^{\circ}\right) \mathrm{s}$

\subsubsection{Fuzzy Based Non-probabilistic Impact Analysis}

In this sub-section, we present numerical results corresponding to the non-probabilistic assessment based on fuzzy analysis, which is beneficial if the complete description of the probability distribution of the input variables is not available. In this paper, the fuzzy approach is used to find out the
81 81 81 $81 ;$ $81 !$ 


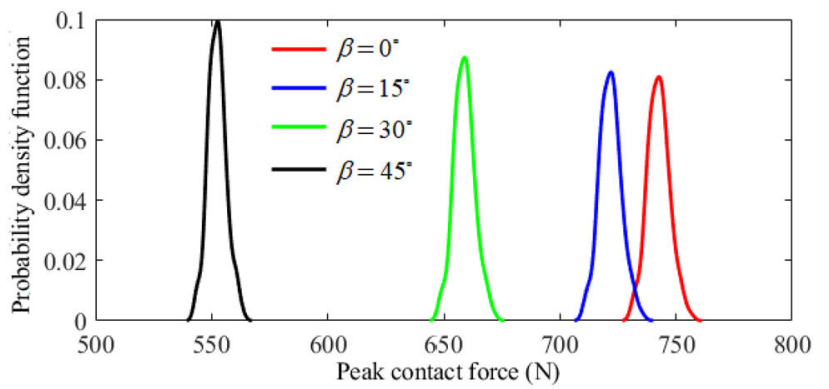

(a)

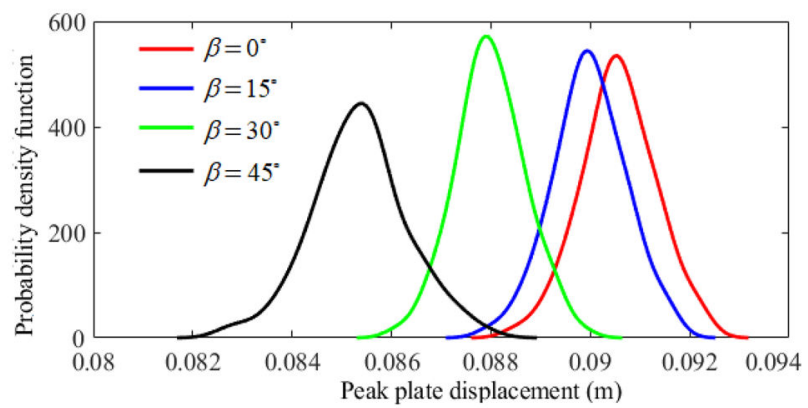

(b)

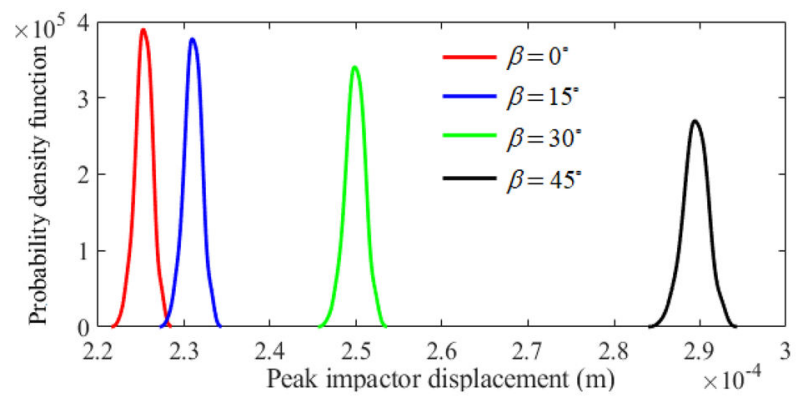

(c)

Fig. 15 Effect of variation of impact angle $(\beta)$ on PDF plots of lowvelocity impact responses considering $t=0.002 \mathrm{~m}, \psi=0^{\circ}, V=5 \mathrm{~m} / \mathrm{s}$, $\rho=0.0085 \frac{N-s}{\mathrm{~cm}^{4}}$, bending stiff laminate $\left(0_{2} \% 30^{\circ}\right) \mathrm{s}$

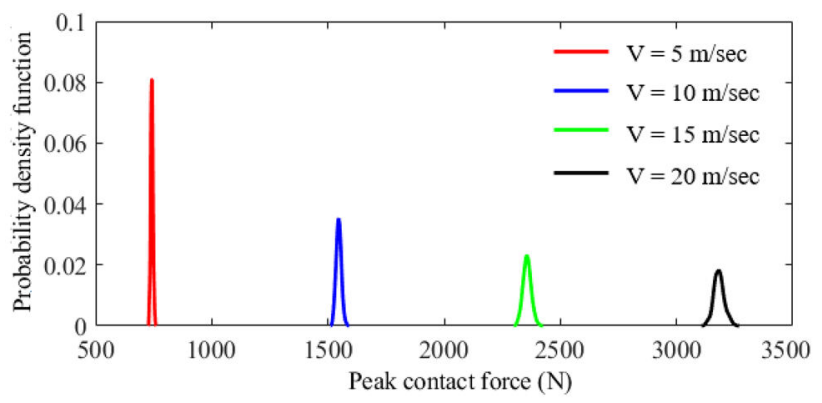

(a)

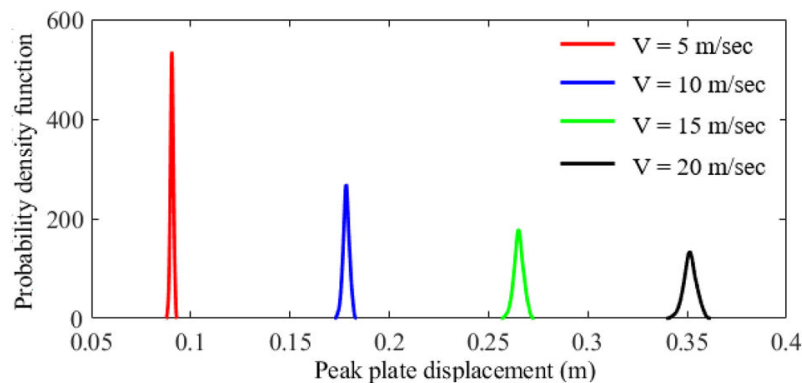

(b)

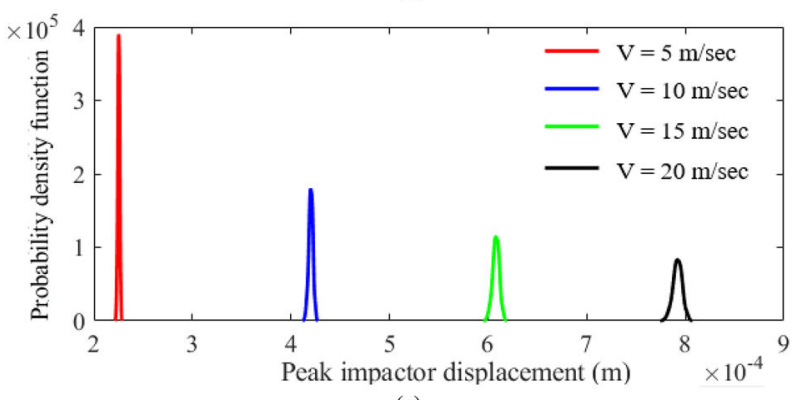

(c)

Fig. 16 Effect of variation of initial velocity of impactor $(V)$ on PDF plots of low-velocity impact responses considering $t=0.002 \mathrm{~m}$, $\psi=0^{\circ}, \beta=0^{\circ}, \rho=0.0085 \frac{N-s}{\mathrm{~cm}^{4}}$, bending stiff laminate $\left(0_{2}^{\circ} / \pm 30^{\circ}\right) \mathrm{s}$

to each level of $\alpha$ - cut. It is found that the resulting output membership functions show a deviation from the triangular distribution of input membership functions.

Similar to the probabilistic analysis, Figs. 21, 22, 23, 24, 25,26 and 27 show the influence of different input variables on the low-velocity impact responses following the fuzzy based approach. In Fig. 21, influence of ply-angle on low velocity impact responses are shown. For torsion stiff 


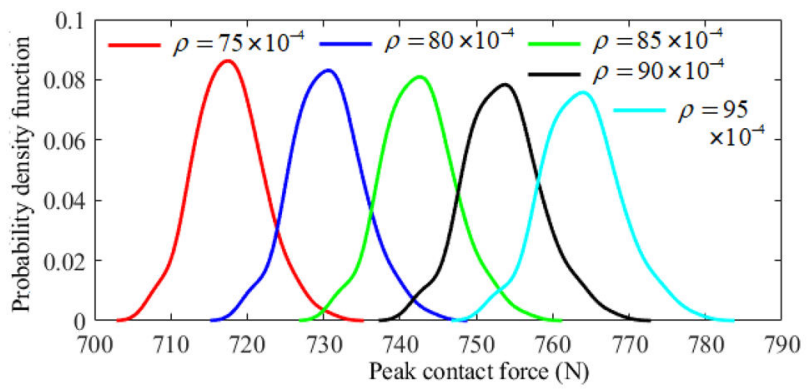

(a)

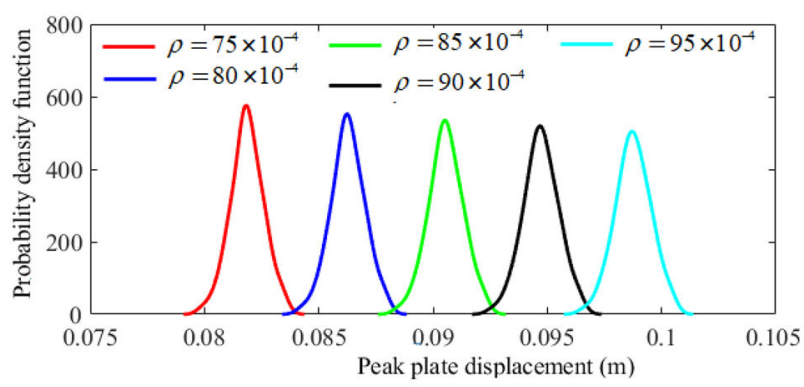

(b)

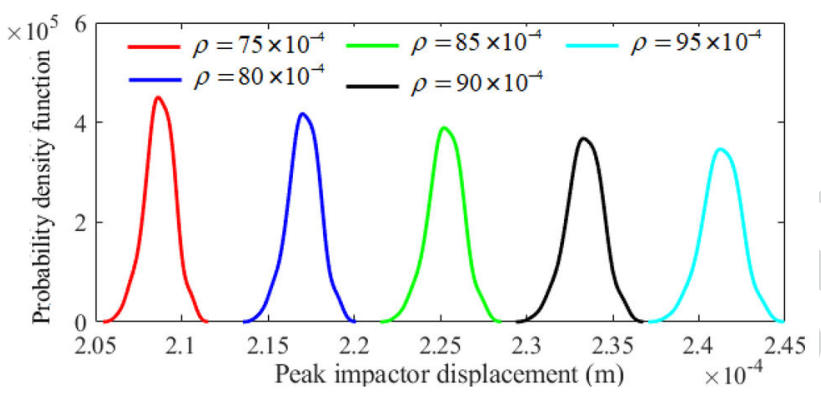

(c)

Fig. 17 Effect of variation of mass density of impactor $\left(\rho\right.$ in $\left.\frac{N-s}{-4}\right)$ on PDF plots of low-velocity impact responses considering $t=0.002 \mathrm{~m}$, $\psi=0^{\circ}, \beta=0^{\circ}, V=5 \mathrm{~m} / \mathrm{s}$, bending stiff laminate $\left(0_{2}^{\circ} / \pm 30^{\circ}\right) \mathrm{s}$

and bending stiff laminate configurations, the maximum and minimum values of contact forces are identified respectively. On the other hand, maximum plate displacement and impactor displacement are observed for bending stiff laminate. Figure 22 shows the effect of the twist angle on fuzzy low velocity impact response behaviour of laminated composite plates. The contact force peak value is noticed to increase

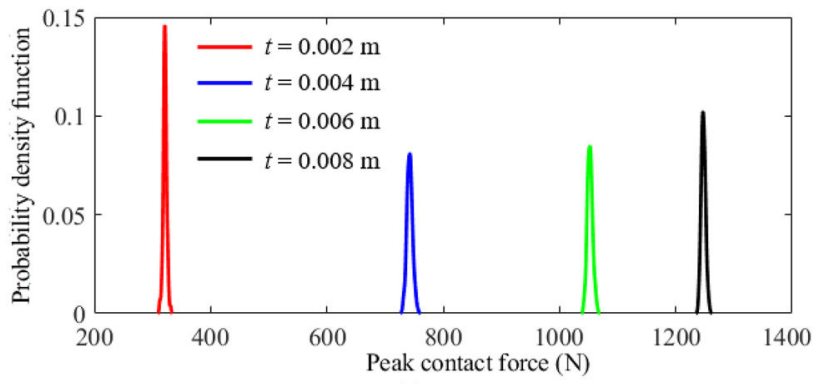

(a)

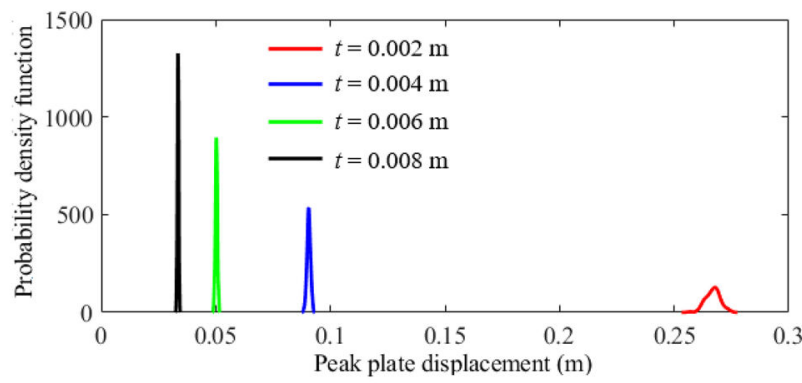

(b)

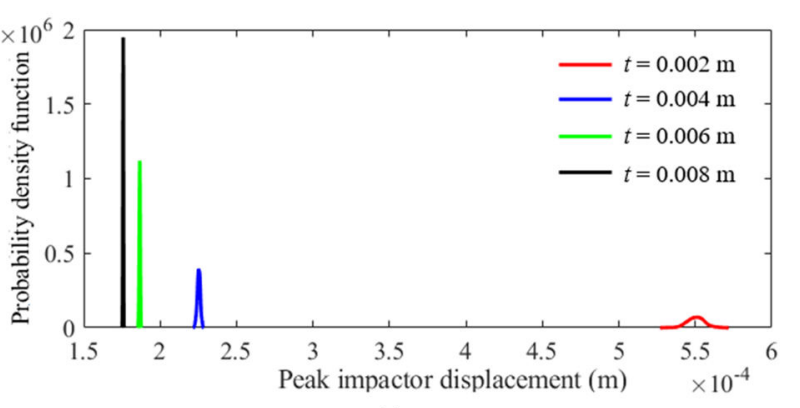

(c)

Fig. 18 Effect of variation of thickness of plate (m) on PDF of lowvelocity impact responses considering $\psi=0^{\circ}, \beta=0^{\circ}, V=5 \mathrm{~m} / \mathrm{s}$, $\rho=0.0085 \frac{N-s}{\mathrm{~cm}^{4}}$, bending stiff laminate $\left(0_{2}{ }^{\circ} / \pm 30^{\circ}\right) \mathrm{s}$

as the angle of twist increases. On the other hand, as the angle of twist increases the plate displacement is found to reduce. The influence of oblique impact angle is shown in Fig. 23. The contact force and plate displacement decrease with the increase in the oblique impact angle; impact displacement is found to have a reverse trend. Figures 24 and 25 show the effect of the mass and initial velocity of the 


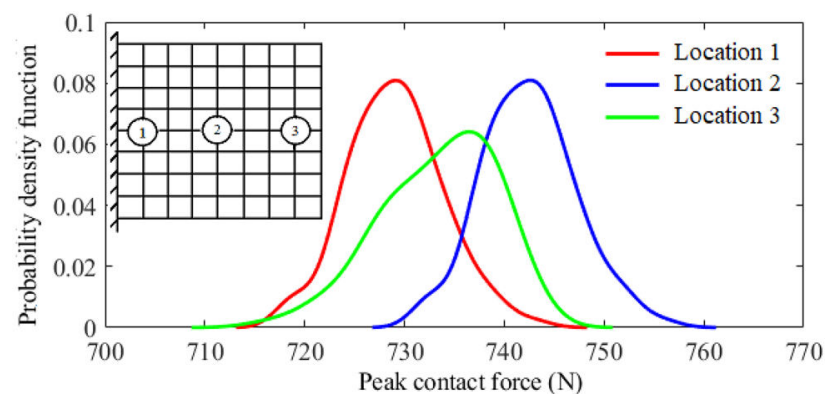

(a)

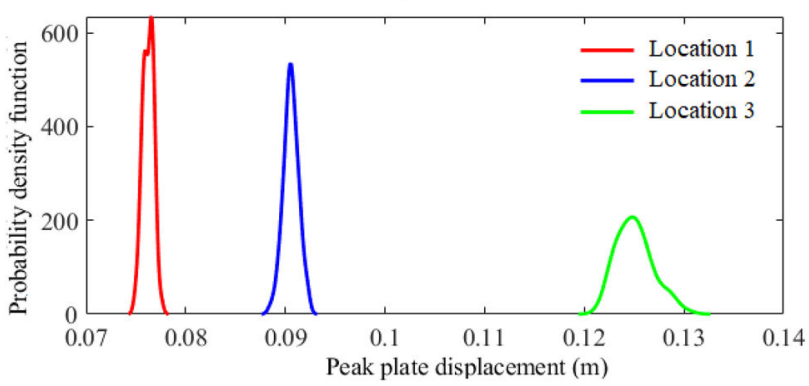

(b)

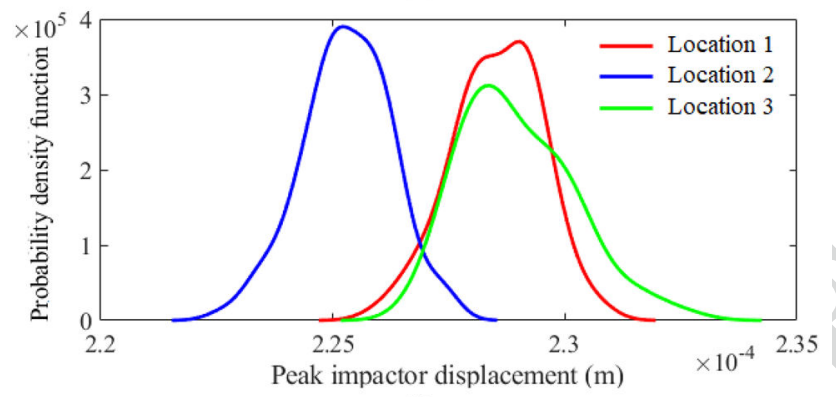

(c)

Fig. 19 Effect of variation of impactor contacting point on PDF plots of low-velocity impact responses considering $t=0.002 \mathrm{~m}, \psi=0^{\circ}$, $\beta=0^{\circ}, V=5 \mathrm{~m} / \mathrm{s}, \rho=0.0085 \frac{N-s}{\mathrm{~cm}^{4}}$, bending stiff laminate $\left(0_{2}^{\circ} / \pm 30^{\circ}\right) \mathrm{s}$ (Location of impact points on the laminated composite plate is indicated in the inset of Fig. 19a)

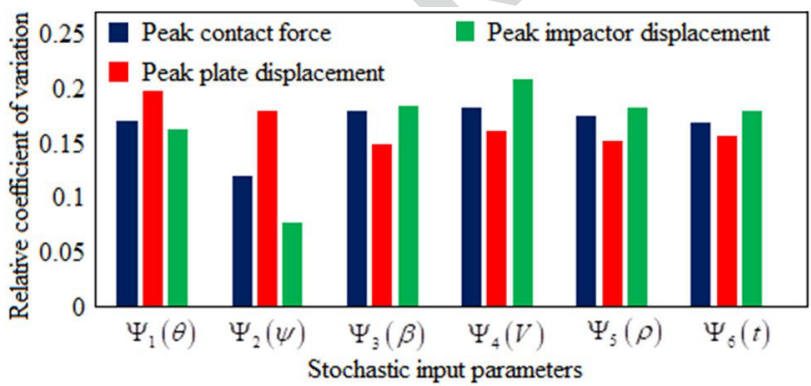

Fig. 20 Relative coefficient of variation (RCOV) for peak contact force, plate displacement, and impactor displacement of centrally impacted glass-epoxy laminated composite plates considering bending stiff laminate $\left(0_{2}^{\circ} / \pm 30^{\circ}\right) \mathrm{s}, \psi=45^{\circ}, \beta=30^{\circ}, V=10 \mathrm{~m} / \mathrm{s}$, $\rho=0.0090 \frac{N-s}{\mathrm{~cm}^{4}}, t=0.004 \mathrm{~m}$ impactor on the transient impact responses, respectively. All the critical responses increase with increase in the impactor mass and initial impactor velocity. The influence of the thickness of plate is shown in Fig. 26, where the contact force increases with the increase in thickness of plate. The plate displacement and impactor displacement are found to decrease with the increase in the thickness of laminate. Finally, Fig. 27 shows the effect of location of impactor on the fuzzy responses considering three different points on the plate surface. The maximum value of contact force is observed when the impact occurs at the centre of the plate. On the other hand, both plate-displacement and impactor displacement are observed to have maximum value when the impact is on location 3 .

\section{Remarks and Perspective on Hybrid Machine Learning Models}

In this paper, we reviewed the possibility of using a hybrid machine learning technique (PC-Kriging) for stochastic computational mechanics considering a critical impact problem. Note that the concept of hybrid machine learning approaches is not new; in fact, there exist a plethora of hybrid machine learning approaches in the literature. The primary idea of these methods is to combine more than one machine learning models so as to exploit the advantages of both (or, all of them). The first use of hybrid machine learning model is perhaps the 'ensemble method' proposed in $[80,81]$. The primary premise of this work was to represent the response as a weighted combination of more than one machine learning techniques. The 'ensemble of surrogate' method has gained significant attention and its application can be found in different domain [82-83]. Analysis of variance (ANOVA) decomposition [84], also known as the high-dimensional model representation (HDMR) [85], is a popular choice among researchers for hybridization. Over the years, researchers have come up with different variants of HDMR/ANOVA by combining it with other machine learning techniques. For example, Shan and Wang [86, 87] combined radial basis function (RBF) with cut-HDMR (aka anchored ANOVA) to formulate RBF-HDMR. Within this framework, the basis functions in cut HDMR are represented by using RBF. In an independent study, Chowdhury et al. [88-90] formulated moving least square based cut-HDMR (MLS-HDMR) for solving structural reliability analysis problems. The formulation for MLS-HDMR and RBF-HDMR are similar; the only difference resides in the 


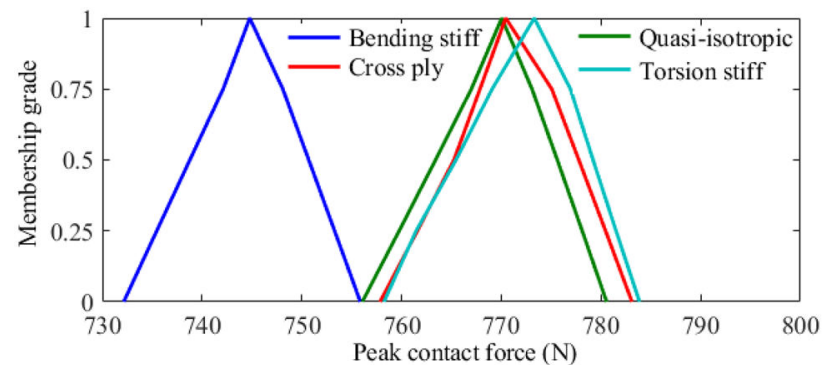

(a)

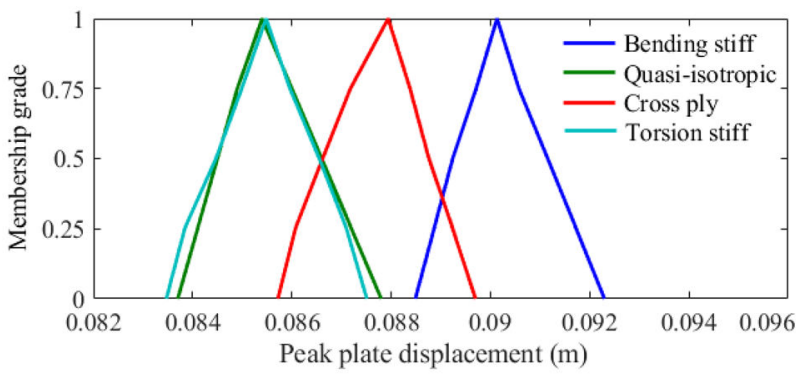

(b)

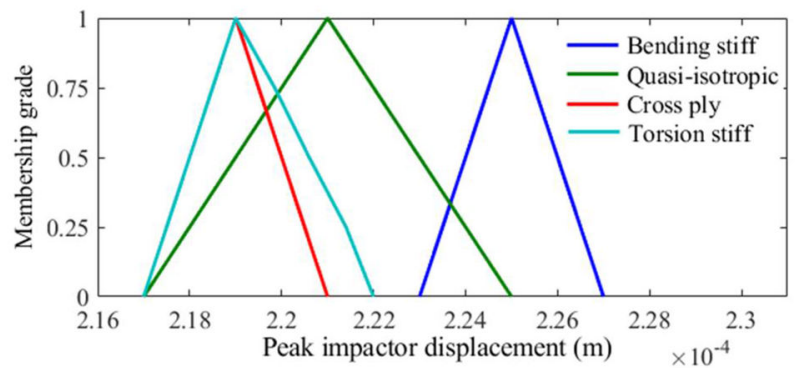

(c)

Fig. 21 Effect of variation of stacking sequence (quasi-isotropic stiff laminate $\left(0^{\circ}, 45^{\circ},-45^{\circ}, 90^{\circ}\right) \mathrm{s}$, torsion stiff laminate $\left(45^{\circ},-45^{\circ}, 45^{\circ}\right.$, $\left.-45^{\circ}\right) \mathrm{s}$, cross ply laminate $\left(90^{\circ}, 0^{\circ}, 90^{\circ}, 0^{\circ}\right) \mathrm{s}$ and bending stiff laminate $\left(0^{\circ}, 0^{\circ}, 30^{\circ},-30^{\circ}\right) \mathrm{s}$ on low-velocity impact responses considering $t=0.002 \mathrm{~m}, \psi=0^{\circ}, \beta=0^{\circ}, V=5 \mathrm{~m} / \mathrm{s}, \rho=0.0085 \frac{N-s}{\mathrm{~cm}^{4}}$ machine learning algorithms discussed above are based on cut-HDMR.

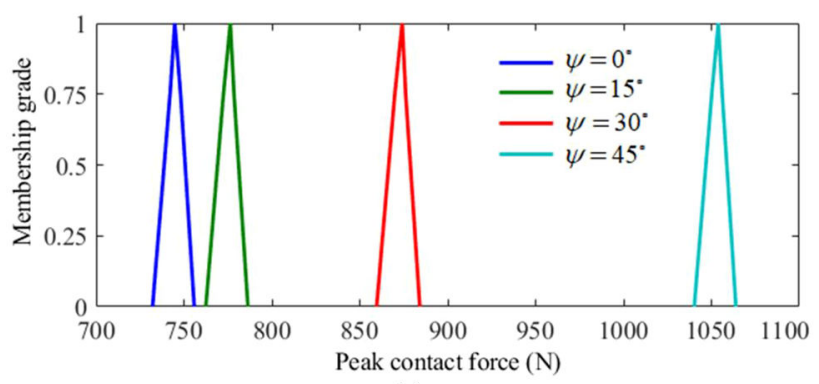

(a)

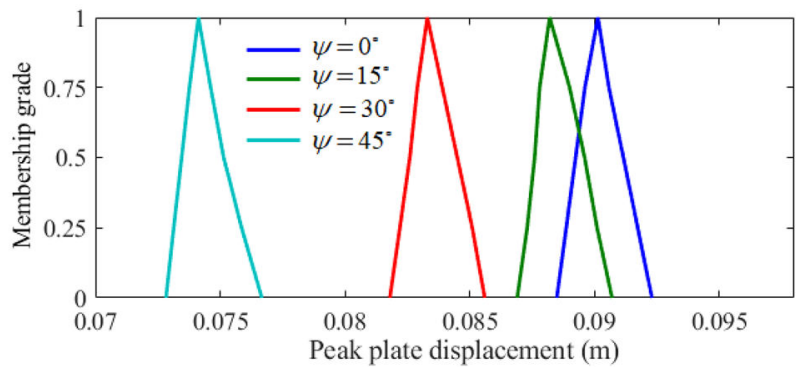

(b)

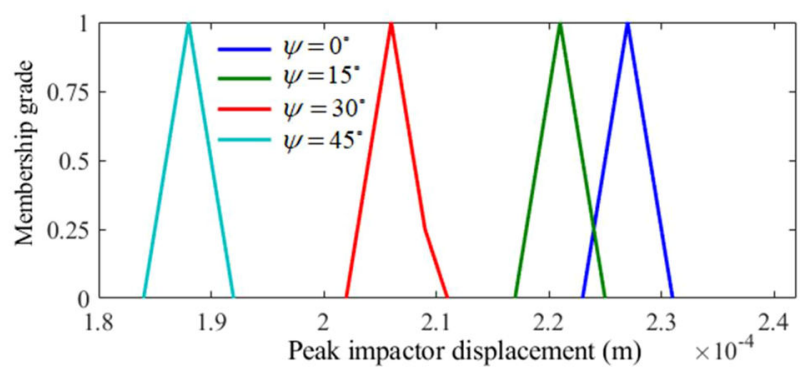

(c)

Fig. 22 Effect of variation of twist angle $(\psi)$ on low-velocity impact responses considering $t=0.002 \mathrm{~m}, \beta=0^{\circ}, V=5 \mathrm{~m} / \mathrm{s}, \rho=0.0085 \frac{N-s}{\mathrm{~cm}^{4}}$, bending stiff laminate $\left(0_{2} \% \pm 30^{\circ}\right) \mathrm{s}$

Hybrid machine learning approaches based on random sampling HDMR, also known as the ANOVA decomposition, can also be found in the literature. Chakraborty and Chowdhury [92] developed a sequential experimental design based generalized ANOVA by coupling polynomial chaos expansion [23, 25, 43] with RS-HDMR [93, 94]. An adaptive version of this algorithm was also proposed [95]. Later 


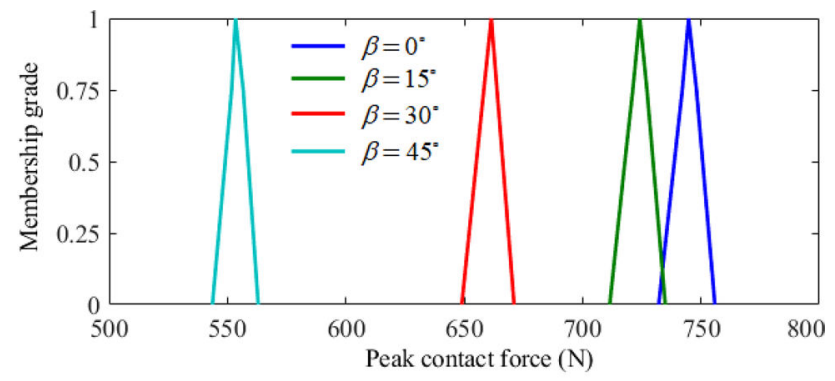

(a)

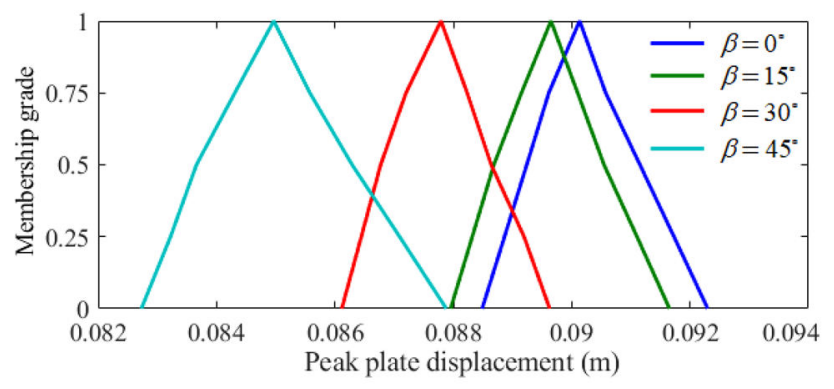

(b)

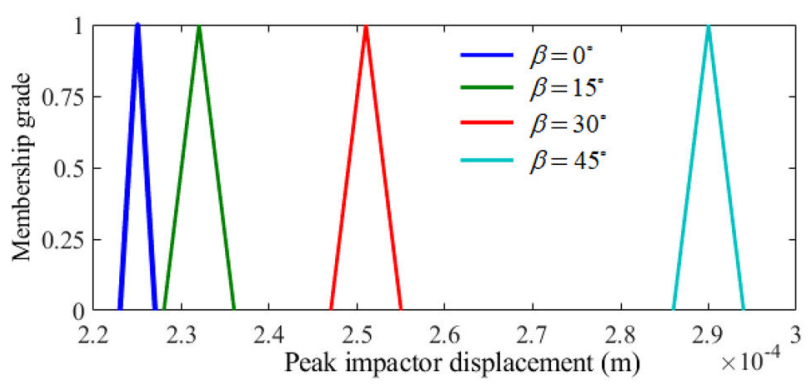

(c)

Fig. 23 Effect of variation of impact angle $(\beta)$ on low-velocity impact responses considering $t=0.002 \mathrm{~m}, \psi=0^{\circ}, V=5 \mathrm{~m} / \mathrm{s}, \rho=0.0085 \frac{N-s}{\mathrm{~cm}^{4}}$, bending stiff laminate $\left(0_{2}^{\circ} / \pm 30^{\circ}\right) \mathrm{s}$ generalized ANOVA was further hybridized by coupling Gaussian process [96-98] with it. This was referred to as the hybrid polynomial correlated function expansion (H-PCFE). In essence, H-PCFE is a fusion of three machine learning algorithms, namely PCE, RS-HDMR and Gaussian process $[22,46,47,99]$. The primary idea of H-PCFE is to represent the mean function of Gaussian process by using generalized ANOVA. Adaptive variants of H-PCFE was proposed in $[100,101]$. It was illustrated that with hybridization (or

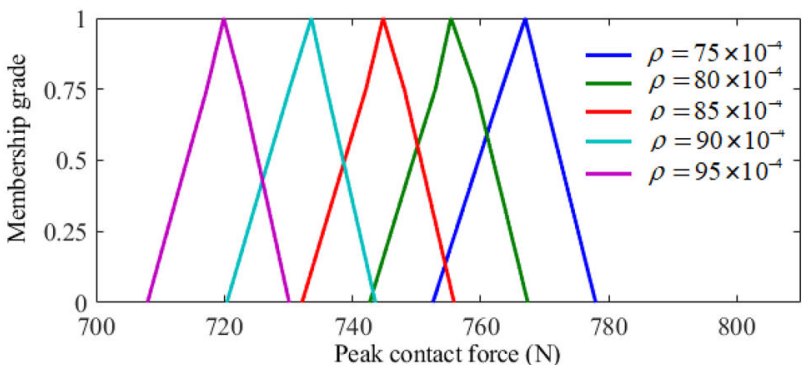

(a)

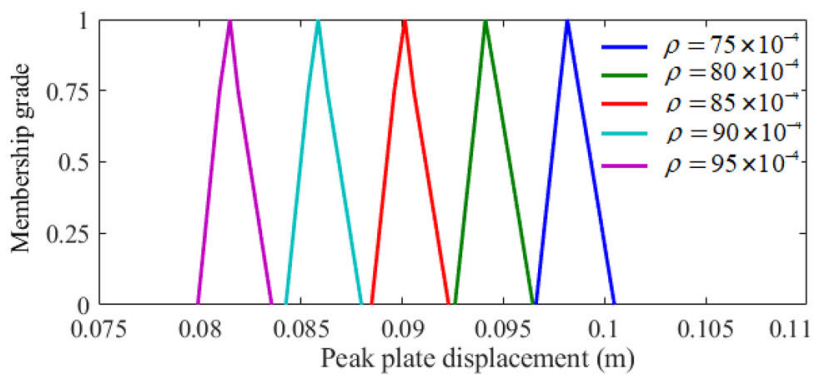

(b)

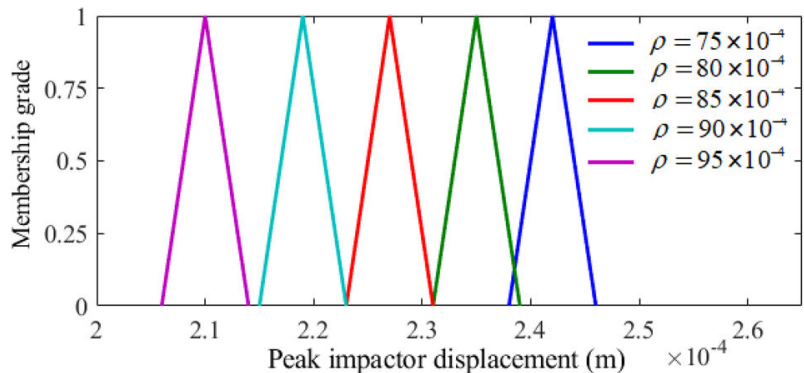

(c)

Fig. 24 Effect of variation of mass density of impactor $\left(\rho\right.$ in $\left.\frac{N-s}{\mathrm{~cm}^{4}}\right)$ on low-velocity impact responses considering $t=0.002 \mathrm{~m}, \psi=0^{\circ}, \stackrel{\mathrm{cm}}{\beta}=0^{\circ}$, $V=5 \mathrm{~m} / \mathrm{s}$, bending stiff laminate $\left(0_{2}^{\circ} / \pm 30^{\circ}\right) \mathrm{s}$

fusion), the accuracy of the machine learning algorithm improves.

Apart from HDMR, hybrid machine learning algorithms based on Gaussian process, also known as the Kriging [20, $21,62]$ is also popular in the literature. In [102], a new hybrid machine learning algorithm was developed by combining fuzzy logic, artificial neural network and Kriging. In another work, Pang et al. [103] combined Gaussian process with neural network. The two methods differ in how 


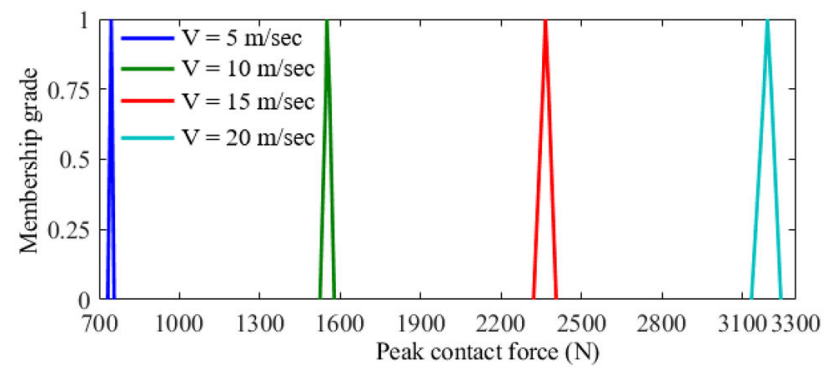

(a)

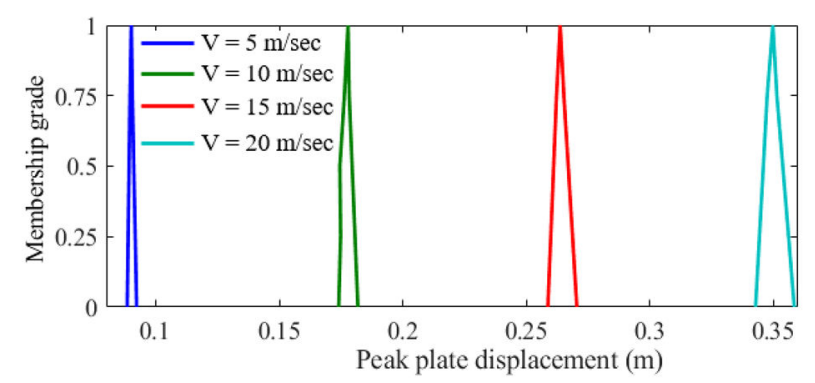

(b)

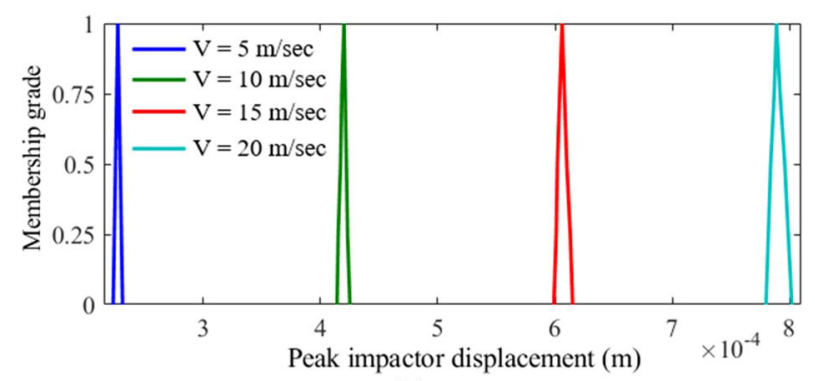

(c)

Fig. 25 Effect of variation of initial velocity of impactor $(V$ in $\mathrm{m} / \mathrm{s})$ on low-velocity impact responses considering $t=0.002 \mathrm{~m}, \psi=0^{\circ}, \beta=0^{\circ}$, $\rho=0.0085 \frac{N-s}{\mathrm{~cm}^{4}}$, bending stiff laminate $\left(0_{2}^{\circ} / \pm 30^{\circ}\right) \mathrm{s}$

the neural network and Gaussian processes are combined. While the former uses neural network and Gaussian process sequentially, separated by fuzzy logic, the latter uses neural network to represent the covariance function of the Gaussian process. The method used in the current paper is also a hybrid machine learning technique, referred to as the polynomial chaos based Kriging (PC-Kriging). This method was

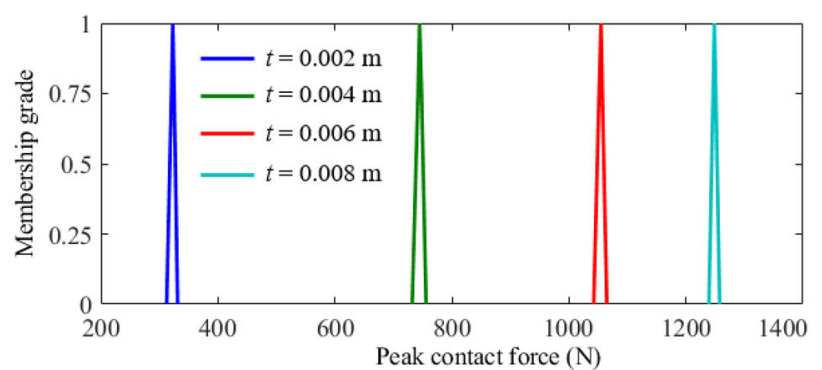

(a)

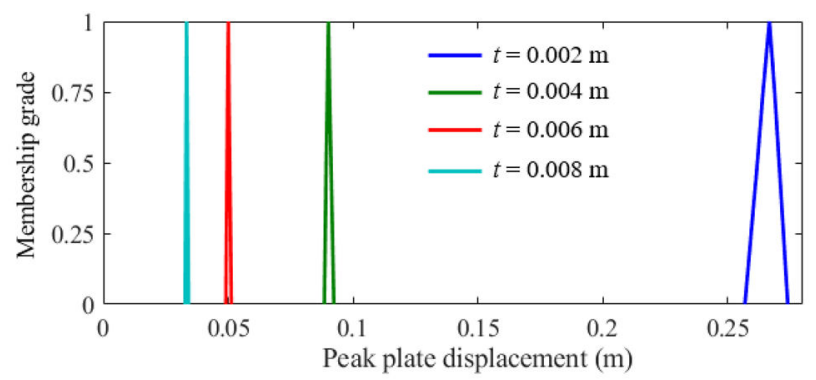

(b)

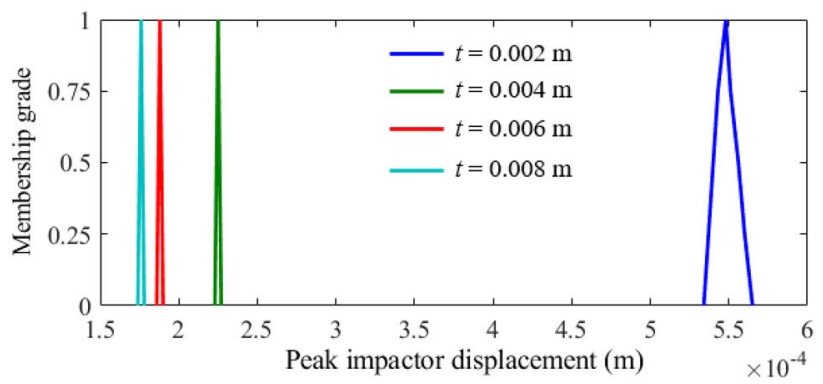

(c)

Fig. 26 Effect of variation of plate thickness $(t)$ on low-velocity impact responses considering $\psi=0^{\circ}, \beta=0^{\circ}, V=5 \mathrm{~m} / \mathrm{s}, \rho=0.0085$ $\frac{N-s}{\mathrm{~cm}^{4}}$, bending stiff laminate $\left(0_{2}^{\circ} / \pm 30^{\circ}\right) \mathrm{s}$

first proposed in [30] and was then further improved in [31]. In this method, the mean function of Kriging is represented by using polynomial chaos expansion. It is argued that polynomial chaos expansion performs a global approximation by using basis function and Kriging performs local approximation by using the covariance kernel. 


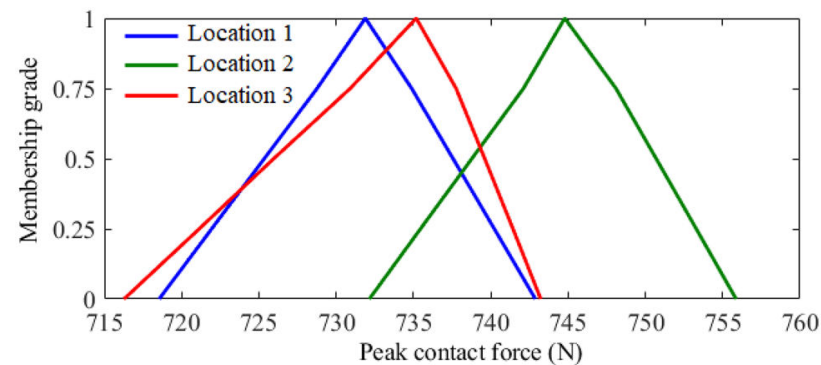

(a)

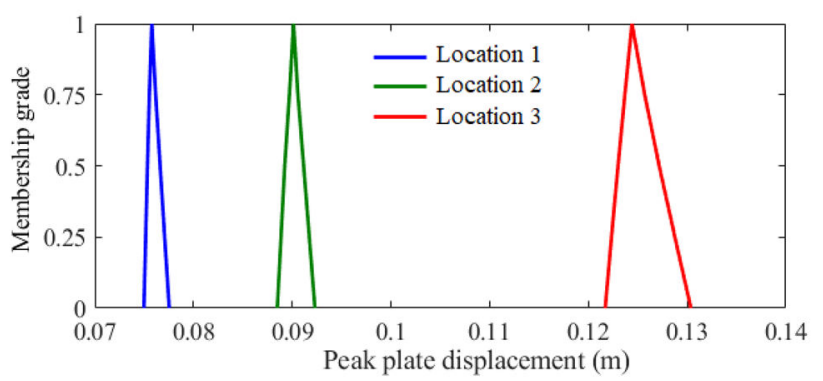

(b)

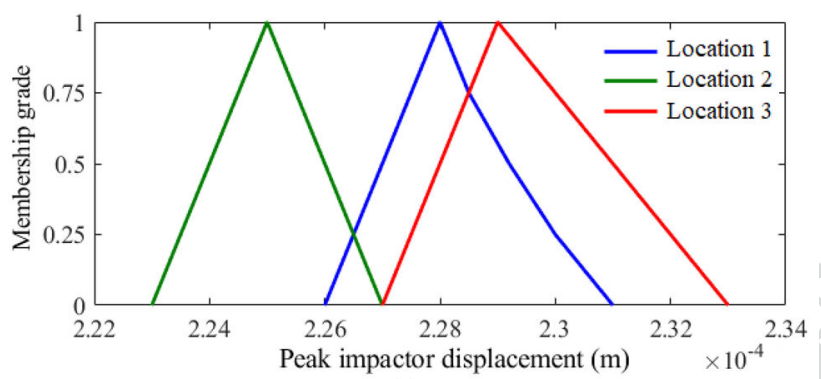

(c)

Fig. 27 Effect of variation of impactor contacting point on lowvelocity impact responses considering $t=0.002 \mathrm{~m}, \psi=0^{\circ}, \beta=0^{\circ}$, $V=5 \mathrm{~m} / \mathrm{s}, \rho=0.0085 \frac{N-s}{\mathrm{~cm}^{4}}$, bending stiff laminate $\left(0_{2}{ }^{\circ} \pm 30^{\circ}\right) \mathrm{s}$ (Location of impact points on the laminated composite plate is indicated in the inset of Fig. 19a)
Based on the literature and the results presented in this paper, it is safe to conclude that hybrid machine learning approaches are generally more accurate as compared to a single machine learning approach (note that such single machine learning approaches have been shown to predict accurately in various engineering problems [104-133]). However, there is no free lunch and this enhancement in the accuracy normally comes at a cost of the efficiency. For instance, H-PCFE discussed above is more accurate but less efficient as compared to the generalized ANOVA. Similarly, polynomial chaos based Kriging used in this paper is more accurate than polynomial chaos and Kriging; however, the computational time necessary for training a polynomial chaos based Kriging is more. To address this issue, researchers over the last few years have developed different adaptive algorithms. Having said that, there is still a significant scope for further developments when it comes to hybrid machine learning algorithms.

\section{Conclusions}

This paper deals with the effects of input-uncertainty on low-velocity impact responses of composite laminates, which is investigated based on an efficient machine learning algorithm. The Newmark's time integration scheme is implemented to solve time-histories of transient responses, while the modified Hertzian contact law is employed to obtain the contact force and other parameters. First, a deterministic analysis is carried to investigate the effects of different system parameters (such as stacking sequence, twist angle, impact angle, initial velocity of impactor, mass density of impactor and thickness of plate). Subsequently a probabilistic analysis is presented to characterize the complete probabilistic descriptions of low-velocity impact responses. Finally, to address the scenario where complete statistical descriptions of the input data are not available (sparse input data), a fuzzy based non-probabilistic approach is presented for low-velocity impact analysis of composites. Since conventional methods for probabilistic and non-probabilistic analyses are exorbitantly computationally expensive, we integrated a hybrid polynomial chaos based kriging (PCKriging) approach with the conventional framework to obtain a high level of computational efficiency. By hybridizing the two powerful metamodelling techniques, polynomial chaos and kriging (to capture the global and local behaviour of a system, respectively), it is possible to exploit the complementary advantages of these two models in a single computational framework. In essence, here we have presented a numerical demonstration of the superiority of hybrid machine learning algorithms over individual models in a systematic way including a critical review of the algorithms.

The novelty of this paper lies in characterizing the effect of source-uncertainty on low- velocity impact of composite plates as well as development of the hybrid simulation approach based on PC-Kriging coupled with the finite element model of composite laminates to achieve computational efficiency. We have presented a comprehensive study following both the probabilistic and non-probabilistic approaches that covers every possible scenario of the availability or unavailability of the statistical distributions of the input parameters. The stochastic results (both probabilistic and non-probabilistic) in this paper show that the inevitable effect of uncertainty has significant effect on the critical impact responses of composite laminates. Thus it is important to adopt an inclusive design approach by quantifying the stochastic variation of the global responses to ensure adequate safety and serviceability of the structure under lowvelocity impact. Besides that, the hybrid PC-Kriging based approach adopted in this study to achieve computational efficiency in the expensive and time-consuming process of modelling impact in complex structural forms like composite 
structures can be useful for other computationally intensive problems of structural analyses and mechanics.

Acknowledgements TM and SN acknowledge the initiation grants received from IIT Kanpur and IIT Bombay, respectively. PKK and RC are grateful for the financial support from MHRD, India during the research work. SC acknowledges the support of XSEDE and Center for Research Computing, University of Notre Dame for providing computational resources required for carrying out this work.

Data availability All data used to generate these results is available in the main paper. Further details could be obtained from the corresponding author(s) upon request.

\section{Compliance with Ethical Standards}

Conflict of interest The authors declare that they have no conflict of interest

\section{References}

1. Naskar S (2018) Spatial variability characterisation of laminated composites, University of Aberdeen

2. Xu S, Chen PH (2013) Prediction of low velocity impact damage in carbon/epoxy laminates. Procedia Eng 67:489-496. https:// doi.org/10.1016/j.proeng.2013.12.049

3. Liu J, He W, Xie D, Tao B (2017) The effect of impactor shape on the low-velocity impact behavior of hybrid corrugated core sandwich structures. Compos Part B Eng 111:315-331. https:// doi.org/10.1016/j.compositesb.2016.11.060

4. Jagtap KR, Ghorpade SY, Lal A, Singh BN (2017) Finite element simulation of low velocity impact damage in composite laminates. Mater Today Proc 4:2464-2469. https://doi.org/10.1016/j. matpr.2017.02.098

5. Balasubramani V, Boopathy SR, Vasudevan R (2013) Numerical analysis of low velocity impact on laminated composite plates. Procedia Eng 64:1089-1098. https://doi.org/10.1016/j. proeng.2013.09.187

6. Tan TM, Sun CT (1985) Use of statical indentation laws in the impact analysis of laminated composite plates. J Appl Mech 52:6. https://doi.org/10.1115/1.3169029

7. Sun CT, Chen JK (1985) On the impact of initially stressed composite laminates. J Compos Mater 19:490-504. https://doi. org/10.1177/002199838501900601

8. Richardson MOW, Wisheart MJ (1996) Review of low-velocity impact properties of composite materials. Compos Part A Appl Sci Manuf 27:1123-1131. https://doi.org/10.1016/1359835X(96)00074-7

9. Ahmed A, Wei L (2015) The low velocity impact damage resistance of the composite structures. Rev Adv Mater 40:127-145

10. Yuan Y, Xu C, Xu T, Sun Y, Liu B, Li Y (2017) An analytical model for deformation and damage of rectangular laminated glass under low-velocity impact. Compos Struct 176:833-843. https://doi.org/10.1016/j.compstruct.2017.06.029

11. Zhang J, Zhang X (2015) An efficient approach for predicting low-velocity impact force and damage in composite laminates. Compos Struct 130:85-94. https://doi.org/10.1016/j.compstruct .2015.04.023

12. Feng D, Aymerich F (2014) Finite element modelling of damage induced by low-velocity impact on composite laminates.
Compos Struct 108:161-171. https://doi.org/10.1016/j.comps truct.2013.09.004

13. Maio L, Monaco E, Ricci F, Lecce L (2013) Simulation of low velocity impact on composite laminates with progressive failure analysis. Compos Struct 103:75-85. https://doi.org/10.1016/j. compstruct.2013.02.027

14. Kim E-H, Rim M-S, Lee I, Hwang T-K (2013) Composite damage model based on continuum damage mechanics and low velocity impact analysis of composite plates. Compos Struct 95:123-134. https://doi.org/10.1016/j.compstruct.2012.07.002

15. Lipeng W, Ying Y, Dafang W, Hao W (2008) Low-velocity impact damage analysis of composite laminates using self-adapting delamination element method. Chin J Aeronaut 21:313-319. https://doi.org/10.1016/S1000-9361(08)60041-2

16. Johnson A, Pickett A, Rozycki P (2001) Computational methods for predicting impact damage in composite structures. Compos Sci Technol 61:2183-2192. https://doi.org/10.1016/ S0266-3538(01)00111-7

17. Coutellier D, Walrick JC, Geoffroy P (2006) Presentation of a methodology for delamination detection within laminated structures. Compos Sci Technol 66:837-845. https://doi. org/10.1016/j.compscitech.2004.12.037

18. Jih CJ, Sun CT (1993) Prediction of delamination in composite laminates subjected to low velocity impact. J Compos Mater 27:684-701. https://doi.org/10.1177/002199839302700703

19. Mukhopadhyay T, Chakraborty S, Dey S, Adhikari S, Chowdhury R (2017) A critical assessment of kriging model variants for high-fidelity uncertainty quantification in dynamics of composite shells. Arch Comput Methods Eng 24:495-518. https:// doi.org/10.1007/s11831-016-9178-z

20. Biswas S, Chakraborty S, Chandra S, Ghosh I (2017) Krigingbased approach for estimation of vehicular speed and passenger car units on an urban arterial. J Transp Eng Part A Syst 143:04016013

21. Kaymaz I (2005) Application of Kriging method to structural reliability problems. Struct Saf 27:133-151

22. Nayek R, Chakraborty S, Narasimhan S (2019) A Gaussian process latent force model for joint input-state estimation in linear structural systems. Mech Syst Signal Process 128:497530. https://doi.org/10.1016/j.ymssp.2019.03.048

23. Xiu D, Karniadakis GE (2002) The Wiener-Askey polynomial chaos for stochastic differential equations. SIAM J Sci Comput 24:619-644

24. Blatman G, Sudret B (2010) An adaptive algorithm to build up sparse polynomial chaos expansions for stochastic finite element analysis. Probab Eng Mech 25:183-197

25. Sudret B (2008) Global sensitivity analysis using polynomial chaos expansions. Reliab Eng Syst Saf 93:964-979

26. Chakraborty S, Chowdhury R (2017) Hybrid framework for the estimation of rare failure event probability. J Eng Mech. https ://doi.org/10.1061/(asce)em.1943-7889.0001223

27. Chakraborty S, Goswami S, Rabczuk T (2019) A surrogate assisted adaptive framework for robust topology optimization. Comput Methods Appl Mech Eng 346:63-84. https://doi. org/10.1016/j.cma.2018.11.030

28. Chakraborty S, Chatterjee T, Chowdhury R, Adhikari S (2017) A surrogate based multi- fidelity approach for robust design optimization. Appl Math Model 47:726-744

29. Chakraborty S, Chowdhury R (2016) Polynomial correlated function expansion. https://doi.org/10.4018/978-1-5225-0588-4. ch012

30. Schobi R, Sudret B, Wiart J (2015) Polynomial chaos based Kriging. Int J Uncertain Quantif 5:171-193. https://doi.org/10.1615/ Int.J.UncertaintyQuantification.2015012467

31. Kersaudy P, Sudret B, Varsier N, Picon O, Wiart J (2015) A new surrogate modeling technique combining Kriging and 
polynomial chaos expansions: application to uncertainty analysis in computational dosimetry. J Comput Phys 286:103-117. https ://doi.org/10.1016/j.jcp.2015.01.034

32. Goswami S, Chakraborty S, Chowdhury R, Rabczuk T (2019) Threshold shift method for reliability-based design optimization. http://arxiv.org/abs/1904.11424

33. Naskar S, Sriramula S (2017) Random field based approach for quantifying the spatial variability in composite laminates. In: 20th International conference on composite structures (ICCS20)

34. Dey S, Mukhopadhyay T, Spickenheuer A, Adhikari S, Heinrich $\mathrm{G}$ (2016) Bottom up surrogate based approach for stochastic frequency response analysis of laminated composite plates. Compos Struct 140:712-727

35. Dey S, Karmakar A (2014) Effect of oblique angle on low velocity impact response of delaminated composite conical shells. Proc Inst Mech Eng Part C J Mech Eng Sci 228:2663-2677. https://doi.org/10.1177/0954406214521799

36. Yang S, Sun C (1982) Indentation law for composite laminates. In: Composite materials: testing and design (6th conference), $\mathrm{p}$ 425. https://doi.org/10.1520/stp28494s

37. Bathe KJ (1996) Finite element procedures. Prentice Hall, New Jersey

38. Wiener N (1938) The homogeneous chaos. Am J Math 60:897-936

39. Hampton J, Doostan A (2015) Coherence motivated sampling and convergence analysis of least squares polynomial Chaos regression. Comput Methods Appl Mech Eng 290:73-97

40. Coelho RF, Lebon J, Bouillard P (2011) Hierarchical stochastic metamodels based on moving least squares and polynomial chaos expansion. Struct Multidiscip Optim 43:707-729

41. Madankan R, Singla P, Patra A, Bursik M, Dehn J, Jones M, Pavolonis M, Pitman B, Singh T, Webley P (2012) Polynomial chaos quadrature-based minimum variance approach for source parameters estimation. Procedia Comput Sci 9:1129-1138

42. Zhang Z, El-Moselhy TA, Elfadel IM, Daniel L (2014) Calculation of generalized polynomial-chaos basis functions and Gauss quadrature rules in hierarchical uncertainty quantification. IEEE Trans Comput Des Integr Circuits Syst 33:728-740

43. Blatman G, Sudret B (2011) Adaptive sparse polynomial chaos expansion based on least angle regression. J Comput Phys 230:2345-2367

44. Jacquelin E, Adhikari S, Sinou JJ, Friswell MI (2015) Polynomial chaos expansion in structural dynamics: accelerating the convergence of the first two statistical moment sequences. J Sound Vib 356:144-154

45. Pascual B, Adhikari S (2012) Hybrid perturbation-polynomial chaos approaches to the random algebraic eigenvalue problem. Comput Methods Appl Mech Eng 217-220:153-167

46. Bilionis I, Zabaras N (2012) Multi-output local Gaussian process regression: applications to uncertainty quantification. J Comput Phys 231:5718-5746

47. Bilionis I, Zabaras N, Konomi BA, Lin G (2013) Multi-output separable Gaussian process: towards an efficient, fully Bayesian paradigm for uncertainty quantification. J Comput Phys 241:212-239

48. Krige DG (1951) A statistical approach to some basic mine valuation problems on the witwatersrand. J Chem Metall Min Soc S Afr 52:119-139

49. Krige DG (1951) A statisitcal approach to some mine valuations and allied problems at the Witwatersrand, University of Witwatersrand

50. Olea RA (2011) Optimal contour mapping using Kriging. J Geophys Res 79:695-702

51. Warnes JJ (1986) A sensitivity analysis for universal kriging. Math Geol 18:653-676
52. Joseph VR, Hung Y, Sudjianto A (2008) Blind Kriging: a new method for developing metamodels. J Mech Des 130:031102

53. Hung Y (2011) Penalized blind kriging in computer experiments. Stat Sin 21:1171-1190

54. Couckuyt I, Forrester A, Gorissen D, De Turck F, Dhaene T (2012) Blind Kriging: implementation and performance analysis. Adv Eng Softw 49:1-13

55. Kennedy M, O'Hagan A (2000) Predicting the output from a complex computer code when fast approximations are available. Biometrika 87:1-13

56. Kamiński B (2015) A method for the updating of stochastic kriging metamodels. Eur J Oper Res 247:859-866

57. Qu H, Fu MC (2014) Gradient extrapolated stochastic kriging. ACM Trans Model Comput Simul 24:1-25

58. Wang B, Bai J, Gea HC (2013, Stochastic Kriging for random simulation metamodeling with finite sampling. In: 39th Design automation conference, vol 3B, ASME, p V03BT03A056. https ://doi.org/10.1115/detc2013-13361

59. Rivest M, Marcotte D (2012) Kriging groundwater solute concentrations using flow coordinates and nonstationary covariance functions. J Hydrol 472-473:238-253

60. Putter H, Young GA (2001) On the effect of covariance function estimation on the accuracy of Kriging predictors. Bernoulli 7:421-438

61. BiscayLirio R, Camejo DG, Loubes JM, MuñizAlvarez L (2013) Estimation of covariance functions by a fully data-driven model selection procedure and its application to Kriging spatial interpolation of real rainfall data. Stat Methods Appl 23:149-174

62. Saha A, Chakraborty S, Chandra S, Ghosh I (2018) Kriging based saturation flow models for traffic conditions in Indian cities. Transp Res Part A Policy Pract 118:38-51. https://doi. org/10.1016/j.tra.2018.08.037

63. Sobol IM (1976) Uniformly distributed sequences with an additional uniform property. USSR Comput Math Math Phys $16: 236-242$

64. Bratley P, Fox BL (1988) Implementing Sobol's quasirandom sequence generator. ACM Trans Math Softw 14:88-100

65. Witteveen JAS, Bijl H (2006) Modeling arbitrary uncertainties using gram-schmidt polynomial chaos. In: 44th AIAA aerospace sciences meeting and exhibition, American Institute of Aeronautics and Astronautics, Reston, Virigina. https://doi. org/10.2514/6.2006-896

66. Hanss M, Willner K (2000) A fuzzy arithmetical approach to the solution of finite element problems with uncertain parameters. Mech Res Commun 27:257-272. https://doi.org/10.1016/ S0093-6413(00)00091-4

67. Moens D, Hanss M (2011) Non-probabilistic finite element analysis for parametric uncertainty treatment in applied mechanics: recent advances. Finite Elem Anal Des 47:4-16. https://doi.org/10.1016/j.finel.2010.07.010

68. Kollár LP, Springer GS (2003) Mechanics of composite structures. Cambridge University Press, Cambridge. https://doi. org/10.1017/cbo9780511547140

69. Kalita K, Mukhopadhyay T, Dey P, Haldar S (2020) Genetic programming assisted multi- scale optimization for multiobjective dynamic performance of laminated composites: the advantage of more elementary-level analyses. Neural Comput Appl 32:7969-7993

70. Kumar RR, Mukhopadhyay T, Pandey KM, Dey S (2019) Stochastic buckling analysis of sandwich plates: the importance of higher order modes. Int J Mech Sci 152:630-643

71. Naskar S, Mukhopadhyay T, Sriramula S (2019) Spatially varying fuzzy multi-scale uncertainty propagation in unidirectional fibre reinforced composites. Compos Struct 209:940-967

72. Dey S, Mukhopadhyay T, Naskar S, Dey TK, Chalak HD, Adhikari S (2019) Probabilistic characterization for dynamics 
and stability of laminated soft core sandwich plates. J Sandwich Struct Mater 21(1):366-397

73. Mukhopadhyay T, Naskar S, Karsh PK, Dey S, You Z (2018) Effect of delamination on the stochastic natural frequencies of composite laminates. Compos B Eng 154:242-256

74. Naskar S, Mukhopadhyay T, Sriramula S (2018) Probabilistic micromechanical spatial variability quantification in laminated composites. Compos B Eng 151:291-325

75. Karsh PK, Mukhopadhyay T, Dey S (2019) Stochastic lowvelocity impact on functionally graded plates: probabilistic and non-probabilistic uncertainty quantification. Compos B Eng 159:461-480

76. Karsh PK, Mukhopadhyay T, Chakraborty S, Naskar S, Dey S (2019) A hybrid stochastic sensitivity analysis for lowfrequency vibration and low-velocity impact of functionally graded plates. Compos B Eng 176:107221

77. Kumar RR, Mukhopadhyay T, Naskar S, Pandey KM, Dey S (2019) Stochastic low-velocity impact analysis of sandwich plates including the effects of obliqueness and twist. Thin Walled Struct 145:106411

78. Naskar S, Mukhopadhyay T, Sriramula S (2017) Non-probabilistic analysis of laminated composites based on fuzzy uncertainty quantification. In: 20th International conference on composite structures (ICCS20)

79. Naskar S, Sriramula S (2017) Vibration analysis of hollow circular laminated composite beams: a stochastic approach. In: 12th International conference on structural safety and reliability

80. Goel T, Haftka RT, Shyy W, Queipo NV (2007) Ensemble of surrogates. Struct Multidiscip Optim 33:199-216

81. Müller J, Shoemaker CA (2014) Influence of ensemble surrogate models and sampling strategy on the solution quality of algorithms for computationally expensive black-box global optimization problems. J Glob Optim 60:123-144. https://doi. org/10.1007/s10898-014-0184-0

82. Müller J, Piché R (2011) Mixture surrogate models based on Dempster-Shafer theory for global optimization problems. J Glob Optim 51:79-104. https://doi.org/10.1007/s1089 8-010-9620-y

83. Viana FAC, Haftka RT, Watson LT (2013) Efficient global optimization algorithm assisted by multiple surrogate techniques. J Glob Optim 56:669-689. https://doi.org/10.1007/s 1089 8-012-9892-5

84. Yang X, Choi M, Lin G, Karniadakis GE (2012) Adaptive ANOVA decomposition of stochastic incompressible and compressible flows. J Comput Phys 231:1587-1614

85. Rabitz H, Aliş ÖF (1999) General foundations of high dimensional model representations. J Math Chem 25:197-233

86. Shan S, Wang GG (2010) Survey of modeling and optimization strategies to solve high-dimensional design problems with computationally-expensive black-box functions. Struct Multidiscip Optim 41:219-241. https://doi.org/10.1007/s00158-009-0420-2

87. Shan S, Wang GG (2011) Turning black-box functions into white functions. J Mech Des. https://doi.org/10.1115/1.4002978

88. Chowdhury R, Rao BN (2009) Assessment of high dimensional model representation techniques for reliability analysis. Probab Eng Mech 24:100-115

89. Chowdhury R, Rao BN, Prasad AM (2007) High dimensional model representation for piece-wise continuous function approximation. Commun Numer Methods Eng 24:1587-1609

90. Chowdhury R, Rao BN, Prasad AM (2009) High-dimensional model representation for structural reliability analysis. Commun Numer Methods Eng 25:301-337

91. Huang Z, Qiu H, Zhao M, Cai X, Gao L (2015) An adaptive SVR-HDMR model for approximating high dimensional problems. Eng Comput 32:643-667. https://doi.org/10.1108/ EC-08-2013-0208

92. Chakraborty S, Chowdhury R (2016) Sequential experimental design based generalised ANOVA. J Comput Phys 317:15-32

93. Chakraborty S, Chowdhury R (2017) Polynomial correlated function expansion. In: Modeling and simulation techniques in structural engineering, IGI Global, pp 348-373

94. Chakraborty S, Chowdhury R (2015) Polynomial correlated function expansion for nonlinear stochastic dynamic analysis. J Eng Mech 141:04014132. https://doi.org/10.1061/(ASCE) EM.1943-7889.0000855

95. Chakraborty S, Chowdhury R (2017) Towards 'h-p adaptive' generalized ANOVA. Comput Methods Appl Mech Eng 320:558-581

96. Chakraborty S, Chowdhury R (2016) Moment independent sensitivity analysis: H-PCFE-based approach. J Comput CivEng 31:06016001-1-06016001-11. https://doi.org/10.1061/(asce) cp.1943-5487.0000608

97. Majumder D, Chakraborty S, Chowdhury R (2017) Probabilistic analysis of tunnels: a hybrid polynomial correlated function expansion based approach. Tunn Undergr Space Technol. https ://doi.org/10.1016/j.tust.2017.07.009

98. Chatterjee T, Chakraborty S, Chowdhury R (2016) A bi-level approximation tool for the computation of FRFs in stochastic dynamic systems. Mech Syst Signal Process 70-71:484-505

99. Chakraborty S, Chowdhury R (2019) Graph-theoretic-approachassisted gaussian process for nonlinear stochastic dynamic analysis under generalized loading. J Eng Mech 145:04019105. https ://doi.org/10.1061/(ASCE)EM.1943-7889.0001685

100. Chakraborty S, Chowdhury R (2017) An efficient algorithm for building locally refined hp_adaptive H-PCFE: application to uncertainty quantification. J Comput Phys 351:59-79

101. Chakraborty S, Chowdhury R (2017) Hybrid framework for the estimation of rare failure event probability. J Eng Mech 143:04017010. https://doi.org/10.1061/(ASCE)EM.19437889.0001223

102. Tapoglou E, Karatzas GP, Trichakis IC, Varouchakis EA (2014) A spatio-temporal hybrid neural network-Kriging model for groundwater level simulation. J Hydrol 519:3193-3203. https:// doi.org/10.1016/j.jhydrol.2014.10.040

103. Pang G, Yang L, Karniadakis GE (2019) Neural-net-induced Gaussian process regression for function approximation and PDE solution. J Comput Phys 384:270-288. https://doi.org/10.1016/j. jcp.2019.01.045

104. Dey S, Mukhopadhyay T, Khodaparast HH, Adhikari S (2016) A response surface modelling approach for resonance driven reliability based optimization of composite shells. Periodica Polytechnica Civ Eng 60(1):103-111

105. Naskar S, Mukhopadhyay T, Sriramula S (2018) A comparative assessment of ANN and PNN model for low-frequency stochastic free vibration analysis of composite plates Handbook of probabilistic models for engineers and scientists, Elsevier Publication, pp 527-547

106. Mukhopadhyay T, Dey TK, Dey S, Chakrabarti A (2015) Optimization of fiber reinforced polymer web core bridge deck: a hybrid approach. Struct Eng Int 25(2):173-183

107. Dey S, Mukhopadhyay T, Sahu SK, Adhikari S (2018) Stochastic dynamic stability analysis of composite curved panels subjected to non-uniform partial edge loading. Eur J Mech A Solids 67:108-122

108. Dey S, Mukhopadhyay T, Adhikari S (2017) Metamodel based high-fidelity stochastic analysis of composite laminates: a concise review with critical comparative assessment. Compos Struct 171:227-250

109. Naskar S, Mukhopadhyay T, Sriramula S, Adhikari S (2017) Stochastic natural frequency analysis of damaged thin-walled 
laminated composite beams with uncertainty in micromechanical properties. Compos Struct 160:312-334

110. Dey S, Mukhopadhyay T, Sahu SK, Adhikari S (2016) Effect of cutout on stochastic natural frequency of composite curved panels. Compos B Eng 105:188-202

111. Dey S, Mukhopadhyay T, Spickenheuer A, Gohs U, Adhikari S (2016) Uncertainty quantification in natural frequency of composite plates: an artificial neural network based approach. Adv Compos Lett 25(2):43-48

112. Dey TK, Mukhopadhyay T, Chakrabarti A, Sharma UK (2015) Efficient lightweight design of FRP bridge deck. Proc Inst Civ Eng Struct Build 168(10):697-707

113. Dey S, Mukhopadhyay T, Khodaparast HH, Adhikari S (2016) Fuzzy uncertainty propagation in composites using GramSchmidt polynomial chaos expansion. Appl Math Model 40(7-8):4412-4428

114. Mukhopadhyay T, Naskar S, Dey S, Adhikari S (2016) On quantifying the effect of noise in surrogate based stochastic free vibration analysis of laminated composite shallow shells. Compos Struct 140:798-805

115. Dey S, Naskar S, Mukhopadhyay T, Gohs U, Sriramula S, Adhikari S, Heinrich G (2016) Uncertain natural frequency analysis of composite plates including effect of noise: a polynomial neural network approach. Compos Struct 143:130-142

116. Naskar S, Sriramula S (2018) On quantifying the effect of noise in radial basis based stochastic free vibration analysis of laminated composite beam. In: 8th European conference on composite materials

117. Dey S, Mukhopadhyay T, Khodaparast HH, Kerfriden P, Adhikari S (2015) Rotational and ply-level uncertainty in response of composite shallow conical shells. Compos Struct 131:594-605

118. Mukhopadhyay T, Dey TK, Chowdhury R, Chakrabarti A, Adhikari S (2015) Optimum design of FRP bridge deck: an efficient RS-HDMR based approach. Struct Multidiscip Optim 52(3):459-477

119. Dey S, Mukhopadhyay T, Adhikari S (2018) Uncertainty quantification in laminated composites: a meta-model based approach. CRC Press, Boca Raton

120. Vaishali Mukhopadhyay T, Karsh PK, Basu B, Dey S (2020) Machine learning based stochastic dynamic analysis of functionally graded shells. Compos Struct 237:111870

121. Mukhopadhyay $\mathrm{T}$ (2018) A multivariate adaptive regression splines based damage identification methodology for web core composite bridges including the effect of noise. J Sandwich Struct Mater 20(7):885-903
122. Karsh PK, Mukhopadhyay T, Dey S (2018) Stochastic dynamic analysis of twisted functionally graded plates. Compos B Eng 147:259-278

123. Maharshi K, Mukhopadhyay T, Roy B, Roy L, Dey S (2018) Stochastic dynamic behaviour of hydrodynamic journal bearings including the effect of surface roughness. Int J Mech Sci 142-143:370-383

124. Metya S, Mukhopadhyay T, Adhikari S, Bhattacharya G (2017) System reliability analysis of soil slopes with general slip surfaces using multivariate adaptive regression splines. Comput Geotech 87:212-228

125. Mukhopadhyay T, Mahata A, Dey S, Adhikari S (2016) Probabilistic analysis and design of HCP nanowires: an efficient surrogate based molecular dynamics simulation approach. J Mater Sci Technol 32(12):1345-1351

126. Mukhopadhyay T, Chowdhury R, Chakrabarti A (2016) Structural damage identification: a random sampling-high dimensional model representation approach. Adv Struct Eng 19(6):908-927

127. Mahata A, Mukhopadhyay T, Adhikari S (2016) A polynomial chaos expansion based molecular dynamics study for probabilistic strength analysis of nano-twinned copper. Mater Res Express 3:036501

128. Dey S, Mukhopadhyay T, Sahu SK, Li G, Rabitz H, Adhikari S (2015) Thermal uncertainty quantification in frequency responses of laminated composite plates. Compos B Eng 80:186-197

129. Dey S, Mukhopadhyay T, Khodaparast HH, Adhikari S (2015) Stochastic natural frequency of composite conical shells. Acta Mech 226(8):2537-2553

130. Mukhopadhyay T, Dey TK, Chowdhury R, Chakrabarti A (2015) Structural damage identification using response surface based multi-objective optimization: a comparative study. Arab J Sci Eng 40(4):1027-1044

131. Naskar S, Sriramula S (2017) Effective elastic property of randomly damaged composite laminates, Engineering postgraduate research symposium, Aberdeen, United Kingdom

132. Dey S, Mukhopadhyay T, Adhikari S (2015) Stochastic free vibration analyses of composite doubly curved shells: a Kriging model approach. Compos B Eng 70:99-112

133. Dey S, Mukhopadhyay T, Adhikari S (2015) Stochastic free vibration analysis of angle-ply composite plates: a RS-HDMR approach. Compos Struct 122:526-536

Publisher's Note Springer Nature remains neutral with regard to jurisdictional claims in published maps and institutional affiliations. 